\title{
On the gauge dependence of vacuum transitions at finite temperature
}

\author{
Mathias Garny ${ }^{a}$ and Thomas Konstandin ${ }^{a, b}$ \\ ${ }^{a}$ DESY, \\ Notkestr. 85, 22607 Hamburg, Germany \\ ${ }^{b}$ CERN Theory Division, \\ 1211 Geneva, Switzerland \\ E-mail: Mathias.Garny@desy.de, Thomas.Konstandin@desy.de
}

\begin{abstract}
In principle, observables as for example the sphaleron rate or the tunneling rate in a first-order phase transition are gauge-independent. However, in practice a gauge dependence is introduced in explicit perturbative calculations due to the breakdown of the gradient expansion of the effective action in the symmetric phase. We exemplify the situation using the effective potential of the Abelian Higgs model in the general renormalizable gauge. Still, we find that the quantitative dependence on the gauge choice is small for gauges that are consistent with the perturbative expansion.
\end{abstract}

KEywords: Thermal Field Theory, Spontaneous Symmetry Breaking

ArXiv EPRINT: 1205.3392 


\section{Contents}

1 Introduction $\quad 1$

2 The model 2

3 Nielsen identity in $R_{\xi}$ gauge $\quad 4$

$\begin{array}{lll}4 & \text { The gradient expansion of the Nielsen identity } & 7\end{array}$

5 The effective potential to order $g^{3}$ and $\lambda \quad 8$

6 Gauge independence in the gradient expansion $\quad 15$

$\begin{array}{llr}7 & \text { Thermal tunneling } & 18\end{array}$

8 Sphaleron numerics $\quad 23$

$\begin{array}{lll}9 & \text { Summary } & 25\end{array}$

$\begin{array}{lr}\text { A Feynman rules } & 27\end{array}$

$\begin{array}{ll}\text { B Wave function corrections } & 27\end{array}$

$\begin{array}{ll}\text { C Wall tension } & 31\end{array}$

$\begin{array}{ll}\text { D Integrals } & 34\end{array}$

\section{Introduction}

Functional methods are an indispensable tool in studying the physics of spontaneous symmetry breaking and phase transitions [1-4]. The main advantage in this approach is that the effective action encodes the ground state of the system in a transparent way. On the technical side, it facilitates the resummation of tadpole diagrams and reduces perturbative calculation to the subset of one-particle-irreducible diagrams.

A large drawback of the effective action is however that it is not explicitly gaugeindependent [5-10]. This makes it necessary to distinguish between the gauge dependence that is expected from the one that is introduced by approximation schemes that break the gauge invariance additionally. The first class of gauge dependences is well represented by the Nielsen identities while the second can for example arise from the use of a loop expansion in perturbation theory or from the expansion of the effective action in gradients.

The aim of the present work is to disentangle these different sources of gauge dependence for vacuum transitions [11] at finite temperature in the Abelian Higgs model. After a 
general introduction to the model (section 2) and the Nielsen identities (sections 3 and 4), the effective potential is calculated (section 5). The main focus is hereby on resummation of infrared effects at finite temperature. In this section, it is also explicitly demonstrated that the position of the minimum of the effective potential transforms under changes of the gauge fixing parameter $\xi$ according to the Nielsen identity. Subsequently (section 6), the same is demonstrated for the (off-shell) effective action in the gradient expansion. Finally (sections 7 and 8), the gauge dependence of the tunneling action and the sphaleron energy are discussed. In these cases an additional complication arises, namely the breakdown of the gradient expansion in the symmetric phase. We discuss, to what extend the gauge dependence of these phenomena can be quantified and potentially reduced before we conclude (section 9).

\section{The model}

In the following we lay out the details of the model under consideration. In order to simplify the analysis and to focus on the main impact of the gauge dependence of the effective action, we discuss an Abelian Higgs model. This model has all necessary ingredients that occur in the Standard Model but does not contain fermions or the correct symmetry breaking pattern as observed in Nature. Nevertheless, for the order of perturbation theory we work at these features are not important and the presented arguments can immediately be carried over to the Standard Model case.

The Lagrangian is given by

$$
\mathcal{L}=D_{\mu} \Phi^{*} D^{\mu} \Phi-\frac{1}{4} F_{\mu \nu} F^{\mu \nu}-V\left(\Phi^{*} \Phi\right)
$$

with the scalar potential

$$
V\left(\Phi^{*} \Phi\right)=-\mu^{2} \Phi^{*} \Phi+\frac{\lambda}{4}\left(\Phi^{*} \Phi\right)^{2}=\frac{\lambda}{4}\left(\Phi^{*} \Phi-v^{2}\right)^{2} .
$$

The gauge covariant derivative is given by $D_{\mu} \Phi=\left(\partial_{\mu}-i g A_{\mu}\right) \Phi$, while the field strength tensor reads $F_{\mu \nu}=\partial_{\mu} A_{\nu}-\partial_{\nu} A_{\mu}$.

We are interested in the effective action $\Gamma$ that is obtained $[1,3]$ by Legendre transformation of the generating functional of connected Greens functions $W(j)$

$$
\begin{aligned}
e^{i W(j)} & =\int \mathcal{D} \Phi \mathcal{D} A e^{i \int d^{4} x \mathcal{L}(\Phi)+j \Phi}, \\
\Gamma(\phi) & =W(j)-\left.\int d^{4} x j \phi\right|_{\phi=d W / d j} .
\end{aligned}
$$

This implies after a shift in the integration variable $\Phi \rightarrow \Phi+\phi$

$$
e^{i \Gamma}=\int \mathcal{D} \Phi \mathcal{D} A e^{i \int d^{4} x \mathcal{L}(\Phi+\phi)+j \Phi} .
$$

By construction the field in the shifted theory does not have a vacuum expectation value (vev). Hence, the bi-linear term containing the source $j$ has to cancels all tadpole diagrams 
and beyond this cancellation, the source $j$ has no impact on the effective action. Therefore $\Gamma$ contains only the connected one-particle-irreducible vacuum diagrams in the shifted theory. The effective potential is obtained by restricting the effective action to homogeneous field expectation values $\phi, V_{\text {eff }}=\Gamma / \int d^{4} x$.

For perturbative calculations, the Lagrange density has to be supplemented by a gauge fixing procedure that results in an additional gauge fixing term and a contribution from the Fadeev-Popov ghosts.

A common choice is to use the general Lorentz gauge

$$
\begin{aligned}
\text { Lorentz gauge : } \quad \mathcal{L}_{g f} & =-\frac{1}{2 \xi}\left(\partial_{\mu} A^{\mu}\right)^{2}, \\
\mathcal{L}_{\mathrm{FP}} & =-\bar{c} \square c .
\end{aligned}
$$

In this gauge the Fadeev-Popov ghosts do not couple to the remaining particles, but the Goldstone boson and the unphysical $k^{\mu}$ polarization of the gauge boson mix after spontaneous symmetry breaking. Nevertheless, this problem is removed in the Landau gauge, $\xi \rightarrow 0$.

Another common choice is the $R_{\xi}$-gauge [12]

$$
\begin{aligned}
R_{\xi} \text {-gauge }: \quad \mathcal{L}_{g f} & =-\frac{1}{2 \xi}\left(\partial_{\mu} A^{\mu}+i g \xi\left(\phi^{*} \Phi-\Phi^{*} \phi\right)\right)^{2}, \\
\mathcal{L}_{\mathrm{FP}} & =-\bar{c}\left(\square+\xi g^{2}\left(\phi^{*} \Phi+\Phi^{*} \phi\right)\right) c .
\end{aligned}
$$

The contribution containing the background field $\phi$ is designed to cancel the mixing term between the Goldstone mode and the $k^{\mu}$ polarization of the gauge boson thus partially diagonalizing the propagator structure of the theory. However, having an explicit dependence on the background field in the gauge fixing term leads to some complications.

First of all, in Lorentz-gauge a shift in $\phi$ is equivalent to attaching an external $\Phi$ field to the diagram $[4,13]$ (this follows immediately from inspecting the Feynman rules). Hence the tadpole can be obtained from the derivative of the effective action. In general gauges this only holds in the minimum of the potential (where the tadpole has to vanish) while in Lorentz gauge this is also true away from the extrema of the potential.

One consequence of this fact is that the two-point functions of the Higgs and Goldstone bosons (at vanishing external momentum) can be obtained from the second derivatives of the effective potential. In particular, the Goldstone bosons are massless in the broken

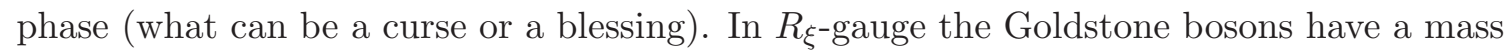
already on tree-level for $\xi \neq 0$.

Furthermore, any tadpole that is generated in a higher loop perturbative calculation can in Lorentz gauge be absorbed by adjusting the appropriate counterterms. This is particularly handy in zero temperature calculations where the Higgs vev is an input observable parameter and kept fixed order by order in the perturbative expansion. Especially when several background fields are present, it is not guaranteed in the $R_{\xi}$-gauge that the tadpoles can be absorbed into the counterterms of the Lagrangian what makes it technically more difficult to keep the vevs fixed in higher loop calculations [13]. Note that at finite 
temperature this feature is not so important, since part of the shift of the vev by loop effects is physical and one has to deal with a temperature-dependent vev anyway.

The behavior of the effective action under gauge transformations was originally analyzed in the Lorentz gauge by Nielsen [5]. Similar relations have been derived shortly after for $R_{\xi}$-gauges [6-8] but their application is somewhat more involved. Still the general picture is the same as in the case of the original Nielsen identities: While the value of the effective action in its extrema is gauge-independent, the position of the extrema can have a gauge dependence. This implies that the critical temperature of the phase transition is gauge-independent. For vacuum transitions, the configurations that extremize the effective action can be gauge-dependent while the value of the action for the configuration is not. The prove of this is sketched in the next section.

Ultimately, we aim to discuss the gauge dependence of the sphaleron energy and the tunneling rate. In this work we focus on the $R_{\xi}$-gauge for two reasons. First, we find it technically easier to eliminate the mixing between the gauge boson and the Goldstone boson and to deal with the peculiarities of the $R_{\xi}$-gauge than to perform loop calculations in the general Lorentz gauge. ${ }^{1}$ Second, the convergence of the perturbation theory is in the Lorentz gauge less obvious than in the $R_{\xi}$-gauge [14-16].

\section{Nielsen identity in $R_{\xi}$ gauge}

In this section we briefly review the Nielsen identities for the effective potential, paying special attention to the additional complications arising in the $R_{\xi}$-gauge. In order to derive the Nielsen identity for a general gauge fixing we write the latter in the form,

$$
\begin{aligned}
\mathcal{L}_{g f} & =-\frac{1}{2 \xi} F^{2}, \\
\mathcal{L}_{\mathrm{FP}} & =-\bar{c}\left(\frac{\delta F}{\delta A_{\mu}} \partial_{\mu}+\frac{\delta F}{\delta \Phi} i g \Phi+\frac{\delta F}{\delta \Phi^{*}}\left(-i g \Phi^{*}\right)\right) c,
\end{aligned}
$$

where $F=\partial_{\mu} A^{\mu}$ in Lorentz gauge and $F=\partial_{\mu} A^{\mu}+i g \xi\left(\tilde{\phi}^{*} \Phi-\Phi^{*} \tilde{\phi}\right)$ for the $R_{\xi}$-gauge. For the derivation of the Nielsen identities, it is convenient to discriminate the field expectation value $\phi \equiv\langle\Phi\rangle$ that appears due to the Legendre transformation of the generating functional from the explicit dependence on the background field in the gauge fixing term, which is denoted by $\tilde{\phi}$ here and can be considered as an external parameter at this stage of the calculation. Both fields will be identified in the end, but for now we distinguish them.

The dependence on $F$ in general leads to a gauge dependence of the effective action $\Gamma$. For an arbitrary change in the gauge fixing term parametrized by $F \rightarrow F+\delta F$ and $\xi \rightarrow \xi+\delta \xi$ the change of the functional $W$ is given by [8]

$$
\delta W=-i \int d^{4} x \int d^{4} y j_{i}\left\langle\delta_{g} \Phi_{i}(x) c(x) \bar{c}(y) \delta^{\prime} F(y)\right\rangle,
$$

where $\delta^{\prime} F \equiv \delta F-F /(2 \xi) \delta \xi$. Furthermore, we collectively denote by $\phi_{i}$ all field expectation values that the effective action depends on, and by $\delta_{g} \Phi_{i}$ the variation of the corresponding

\footnotetext{
${ }^{1}$ See [11] for a zero temperature analysis of the gauge dependence of the effective action in general Lorentz gauge.
} 
field operator under a gauge transformation

$$
\delta_{g} \Phi=i g \Phi, \delta_{g} \Phi^{*}=-i g \Phi^{*}, \delta_{g} A_{\mu}=\partial_{\mu} .
$$

The expectation value of an operator $\langle\mathcal{O}\rangle$ is defined in the usual path integral sense.

The functional $W$ is gauge invariant up to the source term involving $j_{i}$ and the gauge fixing term $\mathcal{L}_{g f}+\mathcal{L}_{\mathrm{FP}}$. The identity (3.2) reflects the fact that a change in the gauge fixing functional can via a gauge transformation shifted into a change of the source term. Now, notice that for any external parameter $\lambda$ one has the relation

$$
\left.\frac{d \Gamma(\phi, \lambda)}{d \lambda}\right|_{\phi=\text { const }}=\left.\frac{d W(j, \lambda)}{d \lambda}\right|_{j=\text { const }},
$$

what can be verified using the definition (2.3). The gauge fixing parameters $\xi$ and $\tilde{\phi}$ are external parameters such that one can translate (3.2) into a relation for $\Gamma$ if the left hand side is understood as being varied with $\phi$ kept fixed, namely

$$
\delta \Gamma=i \int d^{4} x \int d^{4} y \frac{\delta \Gamma}{\delta \phi_{i}(x)}\left\langle\delta_{g} \Phi_{i}(x) c(x) \bar{c}(y) \delta^{\prime} F(y)\right\rangle .
$$

This immediately yields the Nielsen identity for the effective action, which results when only considering a change in the gauge parameter $\xi$,

$$
\xi \frac{\partial \Gamma}{\partial \xi}=-\int d^{4} x\left\{\frac{\delta \Gamma}{\delta A_{\mu}(x)} C_{A^{\mu}}(x)+\frac{\delta \Gamma}{\delta \phi(x)} C_{\phi}(x)+\frac{\delta \Gamma}{\delta \phi^{*}(x)} C_{\phi^{*}}(x)\right\},
$$

where the coefficients, taking a possible $\xi$ dependence of $F$ into account, are given by

$$
\begin{aligned}
C_{A^{\mu}}(x) & =\frac{i}{2} \int d^{4} y\left\langle\partial_{\mu} c(x) \bar{c}(y)(F(y)-2 \xi \partial F(y) / \partial \xi)\right\rangle, \\
C_{\phi}(x) & =\frac{i}{2} \int d^{4} y\langle i g \Phi(x) c(x) \bar{c}(y)(F(y)-2 \xi \partial F(y) / \partial \xi)\rangle, \\
C_{\phi^{*}}(x) & =\frac{i}{2} \int d^{4} y\left\langle\left(-i g \Phi^{*}(x)\right) c(x) \bar{c}(y)(F(y)-2 \xi \partial F(y) / \partial \xi)\right\rangle .
\end{aligned}
$$

For a constant field expectation value, chosen to lie along the real axis $\phi=\phi^{*}$, and vanishing background gauge field $A_{\mu}=0$, one obtains the well-known Nielsen identity for the effective potential,

$$
\xi \frac{\partial V_{\mathrm{eff}}(\phi)}{\partial \xi}+C_{0} \frac{\partial V_{\mathrm{eff}}(\phi)}{\partial \phi}=0
$$

The coefficient $C_{0}$ is obtained when evaluating $C(x) \equiv C_{\phi}+\left.C_{\phi^{*}}\right|_{\phi=\phi^{*}}$ for a constant field expectation value $\phi$. In $R_{\xi}$-gauge, the general expression for $C(x)$ reads

$$
C(x)=\frac{i g}{2 \sqrt{2}} \int d^{4} y\left\langle\bar{c}(x) \chi(x) c(y)\left(\partial_{\mu} A^{\mu}(y)+\sqrt{2} g \xi \tilde{\phi} \chi(y)\right)\right\rangle,
$$

while in Lorentz gauge the second summand is absent. Here we have inserted the decomposition $\Phi=\phi+(h+i \chi) / \sqrt{2}$ where $h$ and $\chi$ denote the Higgs and Goldstone field operators, respectively. Note that the restriction to one real background field and vanishing gauge 
fields can lead to spurious minima in the effective potential but is justified in our class of gauges [6]. This is mostly due to the invariance of the full Lagrangian (including gauge fixing and sources) under the transformation $\Phi \rightarrow \Phi^{*}$ and $A_{\mu} \rightarrow-A_{\mu}$.

For the Lorentz gauge these identities can be used directly when inserting the gauge fixing term $F=\partial_{\mu} A^{\mu}$. In the $R_{\xi}$-gauge, the effective action depends in addition parametrically on the external field $\tilde{\phi}$, i.e. $\Gamma=\Gamma[\phi ; \tilde{\phi}]$. We are ultimately interested in deriving a Nielsen identity for the effective action $\left.\bar{\Gamma}[\phi] \equiv \Gamma[\phi ; \tilde{\phi}]\right|_{\tilde{\phi}=\phi}$. Compared to the Lorentz gauge the complication arises that the field derivatives appearing in eq. (3.6) act only on $\phi$, while the field derivative of $\bar{\Gamma}[\phi]$ contains also a part proportional to the derivative of the effective action with respect to the background field $\tilde{\phi}$. Nevertheless, it is possible to derive a Nielsen identity also for $\bar{\Gamma}[\phi]$. The key observation is that the dependence on the background field $\tilde{\phi}$ enters only via the gauge fixing term [8]. For that reason, the change of the effective action when varying the background field also obeys a Nielsen identity, which can be directly obtained from eq. (3.5),

$$
\frac{\delta \Gamma}{\delta \tilde{\phi}(y)}=i \int d^{4} x \frac{\delta \Gamma}{\delta \phi_{i}(x)}\left\langle\delta_{g} \Phi_{i}(x) c(x) \bar{c}(y) \frac{\partial F(y)}{\partial \tilde{\phi}}\right\rangle .
$$

An analogous relation holds for the derivative with respect to the complex conjugated field. One can use the relation above to express the derivatives of $\bar{\Gamma}$ in terms of the derivatives of $\Gamma$ with respect to the field expectation values $\phi, \phi^{*}$ and $A_{\mu}$,

$$
\frac{\delta \bar{\Gamma}}{\delta \phi_{i}(y)}=\left.\int d^{4} x \sum_{j=A_{\mu}, \phi, \phi^{*}} \mathcal{C}_{i j}(y, x) \frac{\delta \Gamma}{\delta \phi_{j}(x)}\right|_{\tilde{\phi}=\phi},
$$

where

$$
\mathcal{C}_{i j}(y, x)=\delta_{i j} \delta(x-y)+i\left\langle\delta_{g} \Phi_{j}(x) c(x) \bar{c}(y) \frac{\partial F(y)}{\partial \tilde{\phi}_{i}}\right\rangle .
$$

These relations imply that, if the fields fulfill the equation of motions derived from the effective action $\Gamma[\phi ; \tilde{\phi}]$, i.e. $\delta \Gamma / \delta \phi_{j}=0$, then the same field configurations also correspond to a stationary point of the effective action $\bar{\Gamma}[\phi]$. In addition, if $\mathcal{C}$ is invertible, it follows that the effective action $\bar{\Gamma}[\phi]$ also fulfills a Nielsen identity which is of the same form as eq. (3.6) except that the coefficients are replaced by the coefficients $\bar{C}_{i}$ given by

$$
\bar{C}_{i}(y)=\int d^{4} x C_{j}(x)\left(\mathcal{C}^{-1}\right)_{j i}(x, y)
$$

for $i=A_{\mu}, \phi, \phi^{*}$. Concretely, for the case of the $R_{\xi^{-}}$gauge considered here, and assuming a vanishing background gauge field, one has

$$
\begin{aligned}
\mathcal{C}_{i j}= & \delta_{i j} \delta(x-y) \\
& +i g^{2} \xi\left(\begin{array}{cc}
\left\langle\Phi(x) c(x) \bar{c}(y) \Phi^{*}(y)\right\rangle & -\left\langle\Phi^{*}(x) c(x) \bar{c}(y) \Phi^{*}(y)\right\rangle \\
-\langle\Phi(x) c(x) \bar{c}(y) \Phi(y)\rangle & \left\langle\Phi^{*}(x) c(x) \bar{c}(y) \Phi(y)\right\rangle .
\end{array}\right)
\end{aligned}
$$

When considering an expectation value that is constant in space-time, the right-hand side can depend only on the difference of the coordinates, $x-y$, and the equations can be 


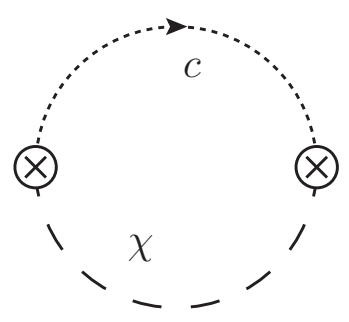

Figure 1. One-loop diagram contributing to the Nielsen coefficient $C_{0}$.

simplified. The resulting Nielsen identity for the effective potential for constant and real field expectation value in $R_{\xi}$-gauge has then the form

$$
\xi \frac{\partial \bar{V}_{\mathrm{eff}}(\phi)}{\partial \xi}+\bar{C}_{0} \frac{\partial \bar{V}_{\mathrm{eff}}(\phi)}{\partial \phi}=0
$$

where $\bar{V}_{\text {eff }}(\phi)=\left.V_{\text {eff }}(\phi, \tilde{\phi})\right|_{\tilde{\phi}=\phi}$, and

$$
\bar{C}_{0}=C_{0}\left(1-i g^{2} \xi \int d^{4}(x-y)\langle\chi(x) c(x) \bar{c}(y) \chi(y)\rangle\right)^{-1} .
$$

The leading contribution arises at one-loop level. For a constant background field, only a single diagram contributes to $C_{0}$ in the $R_{\xi}$-gauge, which can be represented by the diagram shown in Fig 1 . The coefficients $\bar{C}_{0}$ and $C_{0}$ differ only at higher orders, which we do not consider in the following. Therefore, we use the notation $C_{0}$ also in the case of $R_{\xi}$-gauge. Before checking (3.15) explicitly in section 5, we give attention to an analogous identity for the kinetic term in the next section.

\section{The gradient expansion of the Nielsen identity}

As we have just discussed, the effective action contains a certain gauge dependence. Nevertheless, the action evaluated in a saddle point is gauge-independent what is for homogeneous fields reflected by the Nielsen identities. This is explicitly demonstrated in the subsequent section for the broken phase of the effective potential where the convergence of perturbation theory is well under control. However, the sphaleron energy is not a spatially constant configuration and in particular also depends on the effective action evaluated in the symmetric phase. This is problematic, since even though the sphaleron energy is gaugeindependent in principle, a gauge dependence can be introduced due to the breakdown of the gradient expansion. Still, even for configurations that do not fulfill the equations of motion, a gauge-transformation acts on the (off-shell) effective action according to the Nielsen identity. In the following we derive this relation for the effective action in gradient expansion following [11].

To be specific, consider the effective action in gradient expansion

$$
\Gamma=\int d^{4} x\left(Z(\phi) \partial_{\mu} \phi \partial^{\mu} \phi-V_{\mathrm{eff}}(\phi)+\mathcal{O}\left(\partial^{4}\right)\right)
$$


where we introduced a renormalization $Z$ of the kinetic term that in the sphaleron case depends on temperature $T$, the field value $\phi$ and the gauge parameter $\xi$. The coefficient $C(x)$ from the last section that enters the Nielsen identity for the effective action depends functionally on the field value. It can be expanded in field gradients as [11]

$$
C(x)=C_{0}(\phi)+D(\phi) \partial_{\mu} \phi \partial^{\mu} \phi-\partial^{\mu}\left[\tilde{D}(\phi) \partial_{\mu} \phi\right]+\mathcal{O}\left(\partial^{4}\right),
$$

where the coefficients depend on the field value evaluated at position $x$. Note that the term involving $\tilde{D}$ corresponds to a total derivative. Nevertheless it gives rise to a non-zero contribution to the right-hand side of the Nielsen identity for the effective action, and it turns out that it has to be taken into account in a consistent gradient expansion. This term has been neglected in [11] but was not important there at leading order due to the different counting $\lambda \sim g^{4}$. The Nielsen identity is fulfilled when in leading order in gradients the identity (3.15) for the effective potential is established, while in the next order in gradients one finds ${ }^{2}$

$$
\xi \frac{\partial Z}{\partial \xi}=-C_{0} \frac{\partial Z}{\partial \phi}-2 Z \frac{\partial C_{0}}{\partial \phi}+D \frac{\partial V_{\mathrm{eff}}}{\partial \phi}+\tilde{D} \frac{\partial^{2} V_{\mathrm{eff}}}{\partial \phi^{2}}
$$

Notice that this relation cannot ensure the gauge independence of the tunnel action or the sphaleron rate, since the gradient expansion does not apply in these cases, due to $\partial_{\mu} \phi \partial^{\mu} \phi \simeq V_{\text {eff }}$. Still, this relation constitutes an essential check of the consistency of the perturbative scheme that is used to evaluate the effective action. The topic of the subsequent sections is to study this identity in the Abelian Higgs model close to the broken phase explicitly, where perturbative evaluation of all quantities is plausible.

\section{The effective potential to order $g^{3}$ and $\lambda$}

In the following we reproduce the effective potential to order $g^{3}$ and $\lambda$ close to the broken phase. Subsequently, we discuss its gauge dependence and the corresponding Nielsen identity. Before we do so, we briefly motivate why a counting $g^{3} \sim \lambda$ is the appropriate choice in the context of cosmology and finite temperature. It is well known that in the present model the strength of the phase transition crucially depends on cubic contributions to the effective potential of the form $(g \phi)^{3} T$. At the same time the strength of the phase transition is for viable baryogenesis $[17,18]$ constrained by $\phi_{c} / T \sim g^{3} / \lambda>1$. So the largest value of $\lambda$ that is interesting in cosmology is of order $g^{3}$. Besides, for larger values of $\lambda$, the convergence of perturbation theory becomes worse and completely breaks down at $\lambda \sim g^{2}$. For smaller values of $\lambda$, the convergence of the perturbative expansion improves. However, the high temperature expansion that we want to employ in the following would break down since for $\lambda \sim g^{4}$, we find $m_{W} / T \sim g \phi_{c} / T \sim 1$. Therefore, we count in the following $\phi_{c} \sim T$ and $\lambda \sim g^{3}$ as was done in [19]. ${ }^{3}$

\footnotetext{
${ }^{2}$ At finite temperature the correction to the kinetic term can differ in general for the temporal and spatial components. The Nielsen identity can be easily generalized to this case. In the following, $Z$ refers to the correction corresponding to the spatial components.

${ }^{3}$ At zero temperature, the counting $\lambda \sim g^{4}$ is more appropriate $[1,11]$.
} 
At one-loop order the effective potential is given by

$$
V_{\text {eff }}(\phi)=V(\phi)+\frac{1}{2} T^{4} \sum_{i} n_{i} I_{B}\left(m_{i}^{2} / T^{2}\right),
$$

where the sum runs over all species, $n_{i}$ depends on the statistic of the field, and the function $I_{B}$ is given by

$$
\begin{aligned}
I_{B}(y) & =\frac{1}{2 \pi^{2}} \sum_{n} \int d x x^{2} \log \left(4 \pi^{2} n^{2}+x^{2}+y\right) \\
& \simeq \text { const }+\frac{1}{12} y-\frac{1}{6 \pi} y^{3 / 2}+\mathcal{O}\left(y^{2}\right) .
\end{aligned}
$$

Notice that the zero temperature contributions are of order $y^{2}$ and are neglected in the following.

The tree level inverse propagators of the Higgs, Goldstone, gauge fields and ghosts are respectively

$$
\begin{aligned}
i P_{h / \chi / F P}^{-1} & =p^{2}-m_{h / \chi / F P}^{2} \\
i P_{A}^{-1} & =p^{2} g_{\mu \nu}-(1-1 / \xi) p_{\mu} p_{\nu}-m_{A}^{2} g_{\mu \nu},
\end{aligned}
$$

where we have introduced the field-dependent masses

$$
\begin{aligned}
m_{h}^{2} & =\frac{\lambda}{2}\left(3 \phi^{2}-v^{2}\right), \\
m_{\chi}^{2} & =\frac{\lambda}{2}\left(\phi^{2}-v^{2}\right)-2 \xi g^{2} \phi^{2}, \\
m_{A}^{2} & =2 g^{2} \phi^{2}, \\
m_{\mathrm{FP}}^{2} & =2 \xi g^{2} \phi^{2} .
\end{aligned}
$$

These expressions can be used to write the effective potential in the mean field approximation (using the leading terms in (5.2))

$$
\begin{aligned}
V_{\text {mean-field }}=\frac{\lambda}{4}\left(\phi^{2}-v^{2}\right)^{2}+\frac{T^{2}}{24}\left(\frac{\lambda}{2}\left(3 \phi^{2}-v^{2}\right)\right. & +\left[\frac{\lambda}{2}\left(\phi^{2}-v^{2}\right)+2 \xi g^{2} \phi^{2}\right] \\
& \left.+2\left[3 g^{2} \phi^{2}+g^{2} \xi \phi^{2}\right]-4 \xi g^{2} \phi^{2}\right) \\
= & \frac{\lambda}{4}\left(\phi^{2}-v^{2}\right)^{2}+\frac{T^{2}}{24}\left(\lambda\left(2 \phi^{2}-v^{2}\right)+6 g^{2} \phi^{2}\right),
\end{aligned}
$$

where the different contributions are from the Higgs, the Goldstone, the gauge bosons and the ghosts, respectively.

Notice that at tree level and for $\phi=v$, the poles of the Goldstone, the $k^{\mu}$ polarization and the ghosts are all situated at $p^{2}=2 \xi g^{2} \phi^{2}$ and the corresponding contributions to the effective action cancel. ${ }^{4}$ However, at finite temperature the minimum of the effective

\footnotetext{
${ }^{4}$ A term that depends on $\xi$ but not on $\phi$ can be canceled by an appropriate measure for the ghost fields [8].
} 


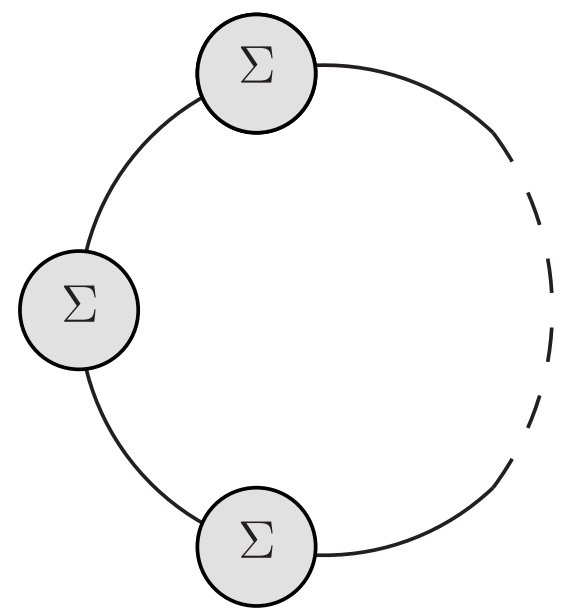

Figure 2. The daisy diagrams that are resummed.
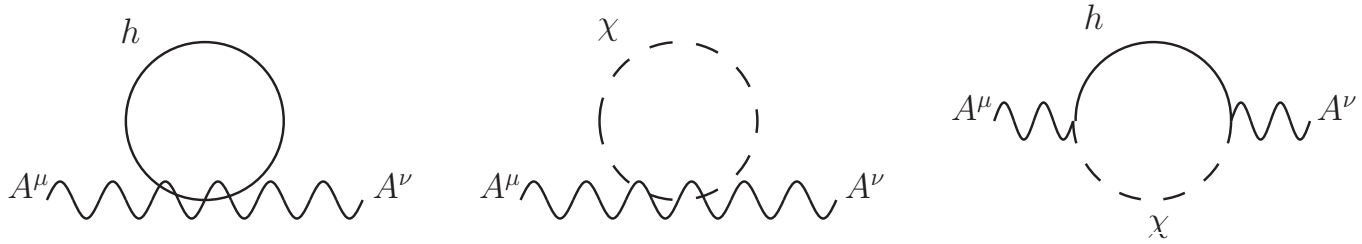

Figure 3. The leading contributions to the self-energy of the gauge bosons.

action moves away from $\phi=v$ and higher loop corrections become important that have to be resummed. In the following we show that these two effects indeed cancel each other in leading order.

The necessity of resummation $[2,21]$ at finite temperature arises because of diagrams as depicted in figure 2. In general, all finite temperature contributions to the self-energies are UV finite. Once the sum over the Matsubara frequencies is performed (or if the real time formalism is used), the integrand contains the particle distribution functions that are exponentially suppressed for momenta larger than the temperature. Hence, the graphs that are apparently UV divergent can be estimated to be of order of the temperature. In particular, tadpole diagrams of the self-energy that arise from the gauge interaction are of order $g^{2} T^{2}$ (e.g. the contributions to the self-energy of the gauge bosons shown in figure 3).

If the particle in the loop has a mass $m_{l}$ and the self-energy is of order $g^{2} T^{2}$, adding self-energies leads to additional factors

$$
\frac{g^{2} T^{2}}{(2 \pi n T)^{2}+p^{2}+m_{l}^{2}}
$$

As long as $n>0$, this yields only a subleading correction of order $g^{2}$. Still, for the zero mode $n=0$, this contribution can be sizable and the according diagrams have to be resummed. This problem is particularly severe when $m_{l}^{2} \ll g^{2} T^{2}$ and the convergence of the perturbative expansion crucially depends on this resummation. This is for example the case 
for the longitudinal gauge bosons, that in the symmetric phase are massless on tree level, but receive self-energy corrections of order $\Sigma \sim g^{2} T^{2}$ at one-loop. But even in the broken phase the self-energy and the mass are both of order $g^{2} \phi^{2}$ and resummation is essential.

In order to resum these diagrams consistently, one has to absorb at least a part $\Sigma_{0}$ of the self-energy into the propagator. In the broken phase, the details how this is done do not really matter as long as

$$
m_{\mathrm{eff}}^{2}=m_{l}^{2}+\Sigma_{0} \gg \Sigma-\Sigma_{0}
$$

This ensures that IR divergences are tamed and the perturbative series converges. These self-energy contributions depend on the temperature and they have to be compensated by introducing a counterterm of equal size in order to avoid a temperature-dependent regularization scheme. These counterterms contribute at higher loop level as we will see below. $\Sigma_{0}$ can in principle depend on momentum and also on the Matsubara number $n$. A particularly simple choice is to only absorb the self-energy of the zero mode in the propagator [19].

For the choice of resummation scheme, it is crucial that in the present context we are only interested in static quantities. If non-static quantities as the plasmon damping rate are considered, the resummation of the zero modes is not enough to ensure the convergence of perturbation theory as discussed in [22]. In this case more elaborate schemes like hard thermal loop resummation have to be employed [23].

In the broken phase (and for $\xi \sim 1$ ), it suffices to absorb the leading term of the self-energy of the gauge bosons. After this is done, the pole structure of all gauge boson propagators is proper in leading order and corrections can be treated perturbatively. On the other hand, the symmetric phase is more problematic because the transverse polarizations remain massless in perturbative calculations.

Within the context of non-Abelian gauge theories, adding additional lines using the self-interaction vertex of the gauge bosons results in a factor $g^{2} T / m_{A}$ what is of order $g$ in the broken phase. In the symmetric phase, on the other hand, the mass of the transverse polarizations is known $[23,24]$ to be non-perturbative and only of order $g^{2} T$ what leads to the well known Linde's problem. For the Abelian case, there is no selfinteraction of gauge bosons and the mass of the transverse polarization should vanish in the symmetric phase [25]. But also here the convergence of the perturbative expansion is not obvious $[24,26]$.

Compared to the unresummed one-loop result in eq. (5.2) resumming only the zero modes gives an additional contribution

$$
T \int \frac{d^{3} p}{(2 \pi)^{3}} \log \frac{p^{2}+m^{2}+\Sigma_{0}}{p^{2}+m^{2}} .
$$

One way to evaluate this expression is to determine its derivative with respect to $m^{2}$ which leads to

$$
T \int \frac{d^{3} p}{(2 \pi)^{3}}\left(\frac{1}{p^{2}+m^{2}+\Sigma_{0}}-\frac{1}{p^{2}+m^{2}}\right)=-\frac{T}{4 \pi}\left(\sqrt{m^{2}+\Sigma_{0}}-\sqrt{m^{2}}\right) .
$$



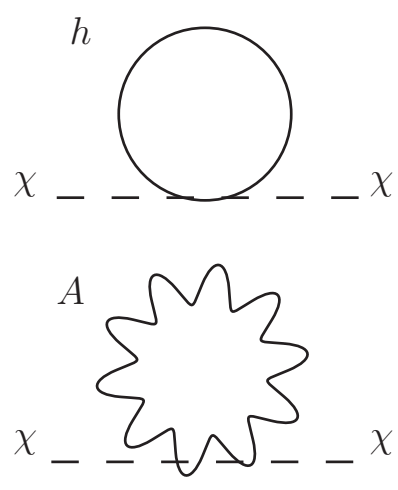
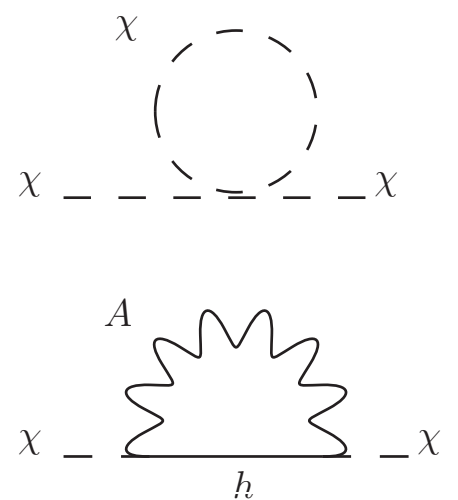

Figure 4. The leading contributions to the self-energy of the Goldstone bosons.

Therefore, the resummation of the zero mode only affects the cubic term in eq. (5.2). The resummed gauge boson masses are given by

$$
\begin{aligned}
& m_{T}^{2}=2 g^{2} \phi^{2}, \\
& m_{L}^{2}=2 g^{2} \phi^{2}+a^{2} g^{2} \phi^{2} .
\end{aligned}
$$

Here, we parametrized the thermal mass of the longitudinal gauge boson with the parameter $a$ because it depends e.g. on the fermionic matter content of the model and is quite different in the Standard Model than in our example calculation.

Now consider the Goldstone bosons that seem to be more interesting than the gauge fields due to the $\xi$ dependence in their mass. The Goldstone bosons are massless on tree level for $\xi=0$, such that resummation can have a large impact on them. If the leading high temperature contributions are taken into account, the self-energy arises from the diagrams in figure 2 and the effective mass is given by

$$
\operatorname{order} g^{2}: \quad m_{\chi, \mathrm{eff}}^{2}=\frac{\lambda}{2}\left(\phi^{2}-v^{2}\right)+2 \xi g^{2} \phi^{2}+\frac{T^{2}}{12}\left(\lambda+3 g^{2}\right) .
$$

This fits nicely with the mean field result in eq. (5.4). If our counting was $\lambda \sim g^{2}$, the mean field result would give the correct leading order result for the effective potential and the above expression shows that the Goldstone boson has a mass $2 \xi g^{2} \phi^{2}$ to this order. This ensures that the cubic terms of the Goldstone boson and the ghost cancel in the broken phase once the next to leading order is taken into account. However, our counting is $\lambda \sim g^{3}$, and to leading order the cubic terms coming from the three physical gauge bosons are important. Even though this term is $\xi$-independent, it induces an additional shift in the vev such that the masses of the Goldstone boson and the ghosts do not coincide any more in the broken minimum of the potential. This problem is particularly severe for small $\xi$, since the ghost becomes massless while the Goldstone boson has a mass of order

$$
m_{\chi, \mathrm{eff}}^{2} \sim \frac{1}{\phi d \phi} T m_{A}^{3} \sim g^{3} T^{2}
$$




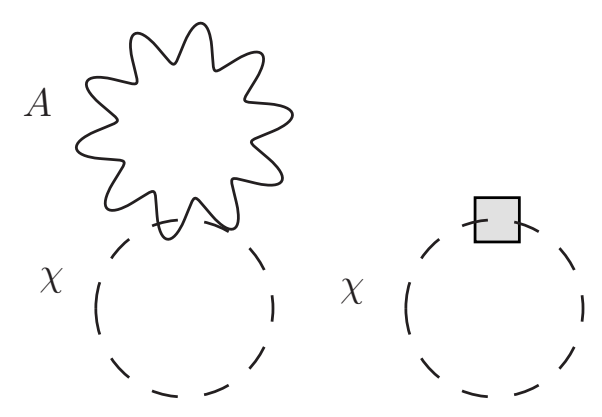

Figure 5. Two-loop contribution to the effective potential. The box denotes the counterterm of the thermal mass.

so the cubic contribution from the Goldstone boson is (partially) screened while the contribution from the remaining ghost is not. This introduces a gauge dependence of order $g^{3}$ into the effective potential.

The solution to this dilemma is that there is a subleading contribution from the gauge bosons to the self-energy of the Goldstone bosons. This is easily seen by realizing that the integral that arises in the tadpole contribution

$$
J_{B}(y)=\frac{1}{2 \pi^{2}} \sum_{n} \int d x x^{2} \frac{1}{4 \pi^{2} n^{2}+x^{2}+y},
$$

is nothing else than the derivative of the one-loop vacuum contribution in eq. (5.2)

$$
J_{B}(y)=\frac{d}{d y} I_{B}(y) .
$$

Hence there is a contribution from the two-loop diagrams [19] depicted in figure 5 that is of order $g^{2} m_{A} T^{3} \sim g^{3} T^{3} \phi$. If $\xi \lesssim g$ this contribution needs to be resummed. This then ensures that the Goldstone boson has the same mass as the ghost in the minimum of the potential to order $g^{3}$ and their cubic contributions cancel each other once they are taken into account. The resummed Goldstone mass is given by

$$
\begin{aligned}
\text { order } g^{3}: \quad m_{\chi, \mathrm{eff}}^{2}= & \frac{\lambda}{2}\left(\phi^{2}-v^{2}\right)+2 \xi g^{2} \phi^{2}+\frac{T^{2}}{12}\left(\lambda+3 g^{2}\right) \\
& -\frac{T g^{2}}{4 \pi}\left(2 m_{T}+m_{L}\right) .
\end{aligned}
$$

In the following, we denote this expression for the Goldstone mass by $m_{\chi}^{2}$.

Finally, we comment on the contribution from the Higgs. Because the Higgs has a mass of order $\lambda \phi^{2}$, its cubic contribution and thermal mass are not relevant to our analysis. Still, there is a subtlety coming from the fact that the loop contribution to the self-energy in figure 6 is of order $\lambda^{2} \phi^{2} T / m_{\chi}$ and diverges in the limit $\xi \rightarrow 0$. This divergence is due to the fact that the self-energy is evaluated at vanishing external momentum. If it is evaluated with external momenta close to the Higgs mass, it is of order $\lambda^{2} \phi^{2} T / k \sim \lambda^{3 / 2} T \phi$ what is small compared to the tree level mass and no resummation is necessary. Anyway, the numerical impact of this contribution is very small as long as $\xi$ is not strictly zero. 


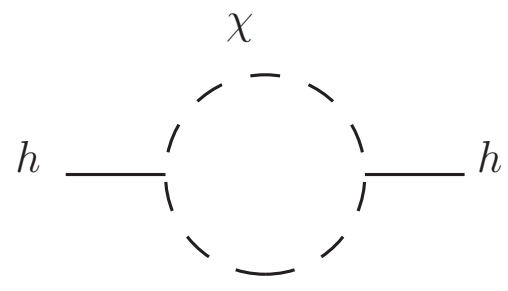

Figure 6. The Goldstone contributions to the self-energy of the Higgs boson.

In conclusion, we arrive to the following expression for the effective potential to order $g^{3}$ and $\lambda$

$$
\begin{aligned}
V_{\left(g^{3}, \lambda\right)}= & \frac{\lambda}{4}\left(\phi^{2}-v^{2}\right)^{2}+\frac{T^{2}}{24}\left(\lambda\left(2 \phi^{2}-v^{2}\right)+6 g^{2} \phi^{2}\right) \\
& -\frac{T}{12 \pi}\left(2 m_{T}^{3}+m_{L}^{3}+m_{\chi}^{3}-m_{\mathrm{FP}}^{3}\right) .
\end{aligned}
$$

We emphasize that the resummed mass of the Goldstone boson is given by (5.14). As argued before, the contribution from the Goldstone boson plus ghost is small when evaluated close to the minimum of the potential. For small $\xi \ll \lambda / g^{2}$ it is of order $\lambda^{3 / 2}$, while for $\xi \sim 1$ one finds

$$
\delta V \sim-\frac{T}{12 \pi}\left(\left(m_{\chi}^{2}\right)^{3 / 2}-\left(m_{\mathrm{FP}}^{2}\right)^{3 / 2}\right) \sim-\frac{T m_{\mathrm{FP}}}{8 \pi}\left(m_{\chi}^{2}-m_{\mathrm{FP}}^{2}\right),
$$

which is of order $g \lambda \sim g^{4}$ and also subleading. However, if it is not neglected, it induces a slight shift in the minimum of order

$$
\delta \phi \simeq \frac{\delta V^{\prime}}{V^{\prime \prime}} \simeq g \sqrt{\xi} \frac{T}{8 \sqrt{2} \pi} .
$$

A shift in the position of the minimum is expected on general grounds, because the field value is not a physical observable. Note that close to the critical temperature this shift is sub-leading,

$$
\delta \phi / \phi_{c} \sim g \sqrt{\xi}
$$

because in our counting $\phi_{c} / T_{c} \sim 1$. Other contributions to the potential of order $g \lambda$ could eventually reduce this shift but actually this shift persists even if higher order contributions are taken into account. This can be seen by inspecting all two-loop diagrams. It turns out that even though some contributions are nominally of order $g^{4}$, they either do not depend on $\xi$ or are not linear in the temperature, so formally they cannot cancel (5.16) completely. However, one expects at higher order a contribution of the form

$$
\frac{1}{2} \delta \phi_{c}^{2} V^{\prime \prime} \simeq \frac{g^{2} \xi \lambda}{512 \pi^{2}} T^{2} \phi_{c}^{2}
$$

to the potential close to the broken phase that removes the remaining gauge dependence of the effective action evaluated in the minimum. That this term is not included in the potential (5.15) introduces a subleading gauge dependence in the critical temperature of order

$$
\frac{\delta T}{T} \simeq \frac{\xi \lambda}{256 \pi^{2}},
$$


what is nominally of order $g^{3}$ and numerically very small.

Let us now discuss to what extent the potential (5.15) respects the Nielsen identity (3.8). First, we show that the shift (5.17) in the position of the minimum is supported by the Nielsen identity. As a function of the parameter $\xi$ and $\phi$, the potential develops iso-potential curves, i.e. there is a function $\bar{\phi}\left(\phi_{0}, \xi\right)$ that fulfills $\bar{\phi}\left(\phi_{0}, \xi_{0}\right)=\phi_{0}$ and according to $(3.8)$

$$
\xi \frac{d}{d \xi} V(\bar{\phi}, \xi)=\xi \frac{d \bar{\phi}}{d \xi} \frac{\partial}{\partial \phi} V+\xi \frac{\partial}{\partial \xi} V=C_{0} \frac{\partial}{\partial \phi} V+\xi \frac{\partial}{\partial \xi} V=0
$$

An explicit expression for the function $C_{0}$ is given in (3.9). The leading contribution obtained by evaluating the diagram shown in figure 1 reads

$$
C_{0} \simeq \frac{1}{2} g^{2} \xi \phi T \int \frac{d^{3} p}{(2 \pi)^{3}} \frac{1}{p^{2}-m_{\chi}^{2}} \frac{1}{p^{2}-m_{\mathrm{FP}}^{2}},
$$

that gives

$$
C_{0} \simeq \frac{1}{8 \pi} \frac{g^{2} \xi \phi T}{m_{\chi}+m_{\mathrm{FP}}} \simeq g \sqrt{\xi} \frac{T}{16 \sqrt{2} \pi},
$$

where the last expression is obtained when evaluating the masses close to the broken phase. This agrees nicely with our findings in (5.17) and the relation $C_{0}=\xi d \bar{\phi} / d \xi$. Using (5.23), it is also possible to verify explicitly that the Nielsen identity for the effective potential (3.8) is indeed satisfied also away from the broken minimum, up to higher order corrections. Namely, taking the $\xi$-derivative of the effective potential given in (5.15) one finds

$$
\xi \frac{\partial V}{\partial \xi}=-\frac{1}{4 \pi} g^{2} \xi \phi^{2} T\left(m_{\chi}-m_{\mathrm{FP}}\right) .
$$

The right-hand side is of higher order close to the minimum where $m_{\chi} \approx m_{\mathrm{FP}}$, such that the value of the effective potential in the minimum is $\xi$-independent, up to higher order corrections, as discussed before. Using that $\frac{\partial V}{\partial \phi} \simeq 2 \phi\left(m_{\chi}^{2}-m_{\mathrm{FP}}^{2}\right)+\mathcal{O}\left(g^{4}, \lambda g\right)$ in combination with (5.23) then shows that the Nielsen identity is respected also away from the broken minimum.

\section{Gauge independence in the gradient expansion}

Let us return to the relation (4.3) that ensures the gauge independence of the effective action in the gradient expansion. An explicit calculation of the wave function correction appearing in the derivative expansion of the effective action yields (see appendix B)

$$
\begin{aligned}
Z=1-\frac{g^{2} T}{3 \pi}\left(\frac{11}{8 m_{T}}-\frac{m_{T}^{2}}{16 m_{L}^{3}}+\frac{2}{m_{\chi}+m_{T}}-\frac{2}{m_{\mathrm{FP}}+m_{T}}\right. \\
\left.+\frac{\xi}{m_{\chi}+m_{\mathrm{FP}}}-\frac{7 \xi}{16 m_{\mathrm{FP}}}-\frac{\xi m_{\mathrm{FP}}^{2}}{16 m_{\chi}^{3}}(1+\delta / \xi)^{2}\right),
\end{aligned}
$$

where we have defined

$$
\delta \equiv \frac{\xi \phi}{2 m_{\mathrm{FP}}^{2}} \frac{\partial}{\partial \phi}\left(m_{\chi}^{2}-m_{\mathrm{FP}}^{2}\right) \simeq \frac{\lambda}{4 g^{2}}-\frac{g^{2} T}{8 \pi}\left(\frac{2}{m_{T}}+\frac{1}{m_{L}}\right) .
$$


Within our counting, $\delta$ is of order $g$ and hence subleading. Still, we keep these corrections since unlike two-loop contributions they are linear in the temperature and (as we shall see) cancel among themselves. The $\xi$ dependence of $Z$ arises via the masses $m_{\chi}$ and $m_{\mathrm{FP}}$, apart from the explicit dependence on $\xi$. Formally, the $\xi$-dependent terms are of the same order as the $\xi$-independent ones. In the limit $\xi \rightarrow 0$, the wave function correction agrees with the one obtained in Landau gauge (see appendix A of [27]).

Note that close to the broken minimum where $m_{\chi} \approx m_{\mathrm{FP}}$, the leading $\xi$-dependent terms cancel and the $\xi$ dependence becomes suppressed. This behavior is precisely the one expected from the Nielsen identity (4.3), because close to the broken minimum all terms on the right-hand side are at most of order $g^{2}$. Namely, the term proportional to $\partial C_{0} / \partial \phi$ is of higher order because close to the broken minimum $C_{0}$ becomes approximately $\phi$-independent. The term involving $C_{0} \partial Z / \partial \phi$ is suppressed because both factors are of order $g$, and the term proportional to the derivative of the effective potential also has to vanish in the broken minimum by definition. Finally, the last term is suppressed as well because the Higgs mass is of order $\lambda \sim g^{3}$. In order to check explicitly that the Nielsen identity (4.3) is also fulfilled for field configurations away from the broken minimum, we have computed the leading contributions to the coefficients $D$ and $\tilde{D}$ contributing to the derivative expansion (4.2) of the Nielsen coefficient $C(x)$ for the full effective action. It is possible to relate these coefficients to Feynman diagrams obtained from attaching one or two external Higgs field lines to the one-loop graph shown in figure 1 that corresponds to the leading contribution to $C(x)$. The diagrams and the explicit expressions are shown in appendix B.

One can check that, with these coefficients (B.16), the result for $Z$ from eq. (6.1), and $C_{0}$ from eq. (5.23), the Nielsen identity (4.3) for the correction of the kinetic term is indeed satisfied to order $g^{2}$. For this calculation, we found it helpful to express also the leading contributions to the field derivatives of $C_{0}$ and $V$ in terms of the Goldstone and ghost masses,

$$
\frac{\partial C_{0}}{\partial \phi} \simeq \frac{g^{2} T \xi}{8 \pi} \frac{m_{\chi}^{2}-m_{\mathrm{FP}}^{2}(1+\delta / \xi)}{m_{\chi}\left(m_{\chi}+m_{\mathrm{FP}}\right)^{2}}
$$

and $\partial V / \partial \phi \simeq 2 \phi\left(m_{\chi}^{2}-m_{\mathrm{FP}}^{2}\right)$, as well as $\partial^{2} V / \partial \phi^{2} \simeq 2\left(m_{\chi}^{2}-m_{\mathrm{FP}}^{2}\right)+4 m_{\mathrm{FP}}^{2} \delta / \xi$ up to corrections of order $\mathcal{O}\left(g^{4}, \lambda g\right)$. Two numerical examples are given in figure 7 .

In conclusion, we have the following findings:

First: the function $C_{0}$ is close to the broken phase of order $g \sqrt{\xi} T$ and hence the $\xi$ dependence of the critical field value $\phi_{c}$ is subleading. Another important consequence is that the first-order nature of the phase transition is a gauge-independent statement $[5,6]$ in the regime where the perturbative expansion converges. ${ }^{5}$

Second: the Nielsen identity (4.3) for $Z$ is in leading order $\left(g^{2}\right)$ fulfilled everywhere. The derivative $\partial C_{0} / \partial \phi$ is of order $g^{2} T / \phi \sqrt{\xi}$. Hence, according to (4.3) $\xi \partial Z / \partial \xi$ can be at most of order $g^{2}$ if $\partial Z / \partial \phi$ is at most of order $g$. From the explicit result (6.1) it turns out that, in the broken phase, indeed $\xi \partial Z / \partial \xi$ is of order $g^{2}$ while $\partial Z / \partial \phi$ is of

\footnotetext{
${ }^{5}$ In our current scheme this also entails the condition that $\xi$ is not much larger than $\mathcal{O}(1)$.
} 

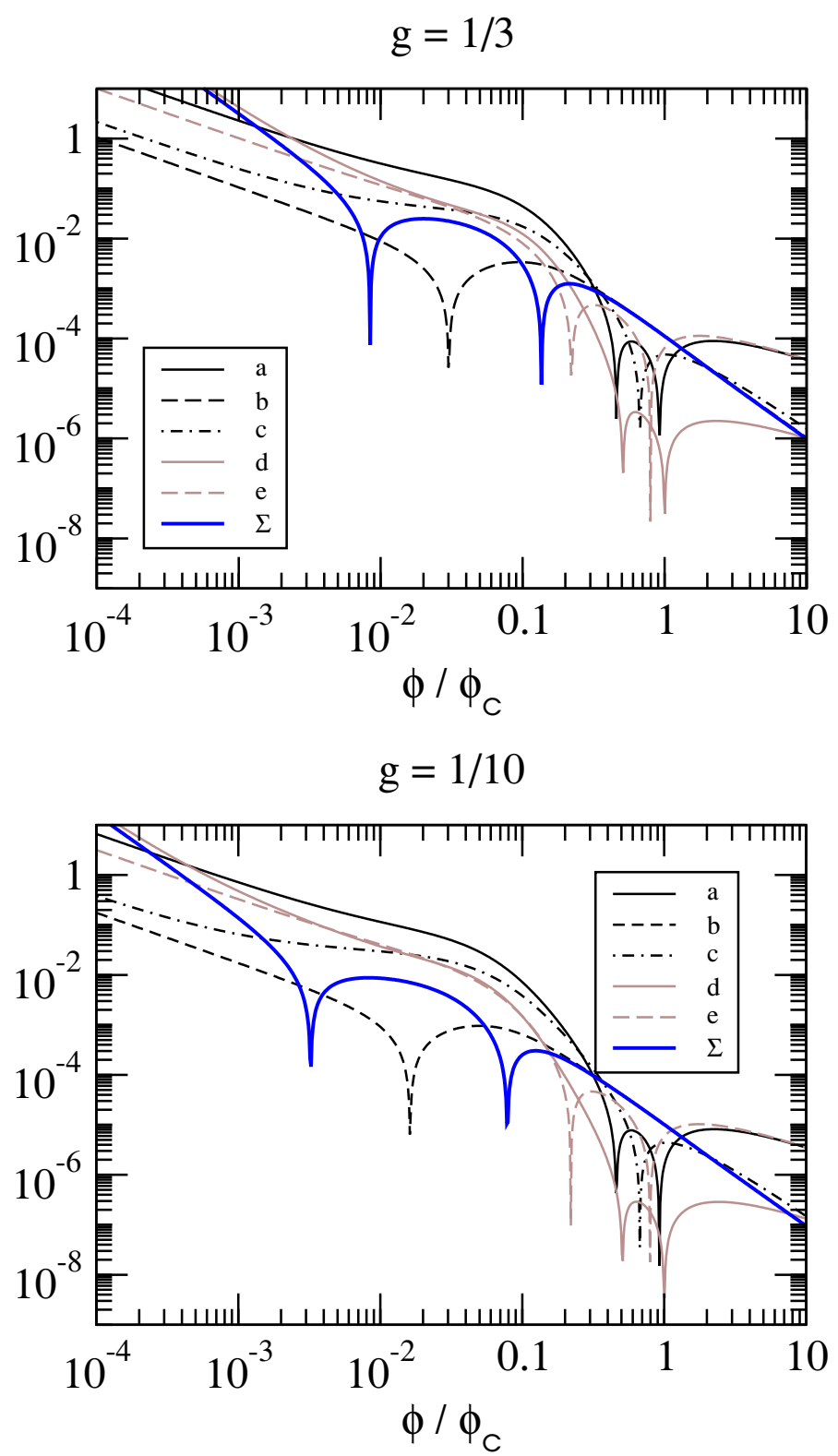

Figure 7. The different contributions to the Nielsen identity (4.3) for $Z(\phi)$ and their sum. The labels (a) to (e) are the five contributions in (4.3) from left to right. The parameters of the upper plot are $g=1 / 3$ and $\lambda=0.015$, while the lower uses $g=1 / 10$ and $\lambda=4.05 \times 10^{-4}$. This ensures $\phi_{c} / T_{c} \sim 1$ in both cases. The gauge parameter is $\xi=0.5$.

order $g$. Finally, $D$ is of order $1 / g$, so all terms in (4.3) are of the same order and their sum to this order vanishes.

Third: even though we cannot check the equation (4.3) in full generality to order $g^{3}$, the one-loop terms are the only contributions to this order that are linear in the temperature. These contributions cancel among themselves. The remaining terms in (4.3) to order $g^{3}$ (denoted by $\Sigma$ in figure 7 ) result from the contributions proportional to $T^{2}$ coming from the product of one-loop terms in $C_{0} d Z / d \phi$. These have to cancel against genuine two-loop contributions that we did not calculate. 
Fourth: close to the symmetric phase the functions $Z, D$ and $\tilde{D}$ diverge what makes the gauge dependence introduced by subleading contributions large. However, this does not indicate a breakdown of perturbation theory. What breaks down is the gradient expansion of the effective action $\Gamma$ and of the function $C$ that was used to arrive at the relation (4.3). In principle, if the gradient expansion was avoided, explicitly gauge-independent results could be obtained. Nevertheless, in practice the gradient expansion is typically used to determine observables like the sphaleron energy or the tunneling rate perturbatively. In the next sections, we discuss to what extend this introduces a gauge dependence to the conventional analysis found in the literature.

\section{Thermal tunneling}

We start the discussion of thermal tunneling by assuming that the gradient expansion of the effective action is sound and only later investigate to what extent this really holds true. The rate of thermal tunneling is mostly determined by the action of the so-called bounce solution of the effective action [28, 29]. The (Euclidean) action with $O(3)$ symmetry is in leading order given by

$$
\Gamma=\frac{4 \pi}{T} \int d \rho \rho^{2}\left(\partial_{\rho} \phi \partial_{\rho} \phi Z(\phi)+V(\phi)\right)
$$

To simplify the discussion, we assume that the temperature is close to the critical temperature where the solutions of the equation of motion is in the 'thin-wall-regime'. In this case, the field only changes in a region $\rho \sim R \gg L$, where $R$ denotes the size of the nucleated bubbles while $L$ denotes the wall thickness of the bounce solution [29]. In this approximation, the action can be rewritten as

$$
\Gamma=\frac{4 \pi}{T} R^{2} \int d \rho\left(\left(\partial_{\rho} \phi\right)^{2} Z(\phi)+V(\phi)\right)-\frac{4 \pi}{3 T} R^{3} \epsilon,
$$

where $\epsilon$ denotes the potential difference between the symmetric and the broken phase. The first integral is invariant under a shift in $\rho$ leading to a conservation of

$$
\left(\partial_{\rho} \phi\right)^{2} Z(\phi)-V(\phi)=\text { const }
$$

For the configuration that dominates the path integral in the tunneling process the constant on the right side vanishes. This gives for the action

$$
\Gamma=\frac{4 \pi}{T} \sigma R^{2}-\frac{4 \pi}{3 T} R^{3} \epsilon
$$

where we defined the wall tension ${ }^{6}$

$$
\sigma=\int d \rho\left(\left(\partial_{\rho} \phi\right)^{2} Z(\phi)+V(\phi)\right)=\int_{0}^{\phi_{c}} d \phi \sqrt{V(\phi) Z(\phi)} .
$$

\footnotetext{
${ }^{6}$ Our definition of the wall tension differs from the usual definition by a factor $\sqrt{2}$ due to our conventions for the kinetic term.
} 
Finally, the bubble size $R$ can be obtained by extremizing this expression, what gives the well-known result [30]

$$
R=\frac{2 \sigma}{\epsilon}, \quad \Gamma=\frac{16 \pi}{3 T} \frac{\sigma^{3}}{\epsilon^{2}}
$$

This little exercise shows that a gauge-independent tunneling rate can in the thin-wall regime only be obtained if the wall tension is gauge-independent (the potential difference $\epsilon$ is obviously gauge-independent due to Nielsen's identity). At first sight, it seems to be impossible that the wall tension is gauge-independent: In the effective potential the gauge dependence starts at relative order $g$, while in $Z$ the gauge dependence first occurs at relative order $g^{2}$. However this argument is not sound, since the gauge dependence arising from the potential is further suppressed in the wall tension. To see this, one can ignore the subleading contributions in $Z$ to order $g^{2}$. This gives then for the wall tension

$$
\xi \frac{d}{d \xi} \sigma \simeq \int_{0}^{\phi_{c}} d \phi \xi \frac{d}{d \xi} \sqrt{V} \simeq \int_{0}^{\phi_{c}} d \phi C_{0} \frac{d}{d \phi} \sqrt{V} \simeq-\int_{0}^{\phi_{c}} d \phi \frac{d C_{0}}{d \phi} \sqrt{V} .
$$

Here we used that the potential vanishes in both phases in the thin-wall approximation. $d C_{0} / d \phi$ is in the broken phase of order $g^{2}$ such that the gauge dependence of the effective potential can in the wall tension cancel against the gauge dependence in $Z$.

Is this result specific to the thin-wall regime and how does this fit together with the statement (4.3)? On first sight, (4.3) only ensure the gauge independence of the action in the gradient expansion, or more specifically as long as $Z \partial_{\mu} \phi \partial^{\mu} \phi \ll V$. On the other hand, for the tunneling bounce solution both terms are of the same order and this expansion does not apply. However, in the present context there is a way to derive a similar relation as (4.3) for the tunneling bounce without this constraint. Starting from (3.2), a functional derivative with respect to $j(x)$ yields

$$
\xi \frac{d \phi(x)}{d \xi}=C(x, \phi(x), \xi),
$$

in case $\phi(x)$ fulfills the equation of motion, $j(x)=0$. So, there is a class of solutions $\varphi(x, \xi)$ with

$$
\left.\frac{d}{d \xi} \Gamma(\phi(x), \xi)\right|_{\phi(x)=\varphi(x, \xi)}=0 .
$$

Using this relation in (7.1) yields

$$
0=4 \pi T \int d \rho \rho^{2}\left(\partial_{\rho} \phi \partial_{\rho} \phi\left[2 \frac{d C}{d \phi}+\frac{d Z}{d \phi} C+\xi \frac{d Z}{d \xi}\right]+\xi \frac{d V}{d \xi}+C \frac{d V}{d \phi}\right)
$$

So qualitatively the same picture emerges as in the thin-wall regime. To relative order $g$, the shift (7.8) in combination with (3.8) ensures the gauge independence but only because $d C / d \phi$ is of order $g^{2}$ while $C$ is of order $g$.

Using the expansion (4.2) in (7.9) then leads to a relation similar to (4.3). Unlike the condition $Z \partial_{\mu} \phi \partial^{\mu} \phi \ll V$, the expansion (4.2) is well justified in the present context at least in the broken phase. Nominally the expansion parameter is $p^{2} / m^{2}$ such that with $p^{2} \phi_{c}^{2} \sim V \sim g^{3} \phi_{c}^{4}$ and masses of order $m^{2} \sim 2 \xi g^{2} \phi^{2}$ the gradient expansion of (4.2) is valid for $\phi \gg \sqrt{g / \xi} \phi_{c}$. In particular, the gradient expansion gets worse for small $\xi$. 
Now notice that in the last section we have shown the relation $\xi d V / d \xi=C_{0} d V / d \phi$ only up to terms of order $g^{5}$ and that $d D / d \phi$ is like $d C_{0} / d \phi$ of order $g^{2}$. Hence to order $g^{5}$, the relation (7.9) is not exactly equivalent to (4.3). In any case, the range of validity is not the same for both relations, since (4.3) is valid for small gradients, while in the derivation of (7.9) we used the equation of motion.

From above discussion, it seems that the gauge dependence of the wall tension is suppressed at least by $g^{2}$ if $Z$ is ignored and that the inclusion of the $Z$ factor might even postpone it to order $g^{3}$. However, the breakdown of the gradient expansion in the symmetric phase prevents a gauge-independent determination of the wall tension in (7.5) to this order. In practice, the expression we obtained for the wave function normalization $Z$ can even become negative for $\phi \sim g \phi_{c}$ such that no reasonable results can be obtained if the leading non-trivial order of $Z$ is taken into account. Also $d C_{0} / d \phi$ is of order $\sqrt{g}$ close to the symmetric phase what indicates that a large gauge dependence arises if the gradient expansion is employed.

The gauge dependence arises mostly from the symmetric phase and integration of eq. (7.7) leads to the estimate

$$
\xi \frac{d \sigma}{d \xi} \simeq \frac{1}{48 \pi} m_{\chi}^{2} T_{c}
$$

for $\xi$ not too small. Here $m_{\chi}$ denotes the Goldstone mass for $\phi=0$, that coincides with the Higgs mass $m_{h}$ in the symmetric phase. Compared with the leading order result

$$
\sigma \simeq \frac{1}{6} m_{\chi} \phi_{c}^{2}
$$

the uncertainty from the gauge dependence scales as $\sqrt{\lambda} \sim g^{3 / 2}$,

$$
\frac{\xi}{\sigma} \frac{d \sigma}{d \xi} \simeq \frac{1}{8 \pi} \frac{m_{\chi} T_{c}}{\phi_{c}^{2}}
$$

This leads to a logarithmic dependence on $\xi$,

$$
\sigma(\xi) / \sigma(1)-1 \propto g^{3 / 2} \ln (\xi),
$$

which nicely fits with our numerical findings shown in figure 8. Especially, this means that for very small values of $\xi$ the gauge dependence becomes enhanced. For $\xi \lesssim g$, the logarithm is ultimately cut off, and the $\xi$ dependence is of the relative order $g^{3 / 2} \ln (g)$. Therefore, it seems that a choice $\xi \gg O(g)$ is slightly preferable for the computation of the wall tension compared to e.g. Landau gauge.

Including the wave function correction $Z$ to the kinetic term in the determination of the wall tension mainly leads to a gauge-independent shift. This shift can be estimated as (details are again given in appendix $\mathrm{C}$ )

$$
\Delta \sigma \simeq-\frac{11 \sqrt{2}}{192 \pi} g m_{\chi} T_{c} \phi_{c}
$$

This scales as $\Delta \sigma / \sigma \sim g$ what is parametrically larger than the gauge-dependent contributions to the wall tension that scale as $g^{3 / 2}$. The gauge dependence of the wall tension 

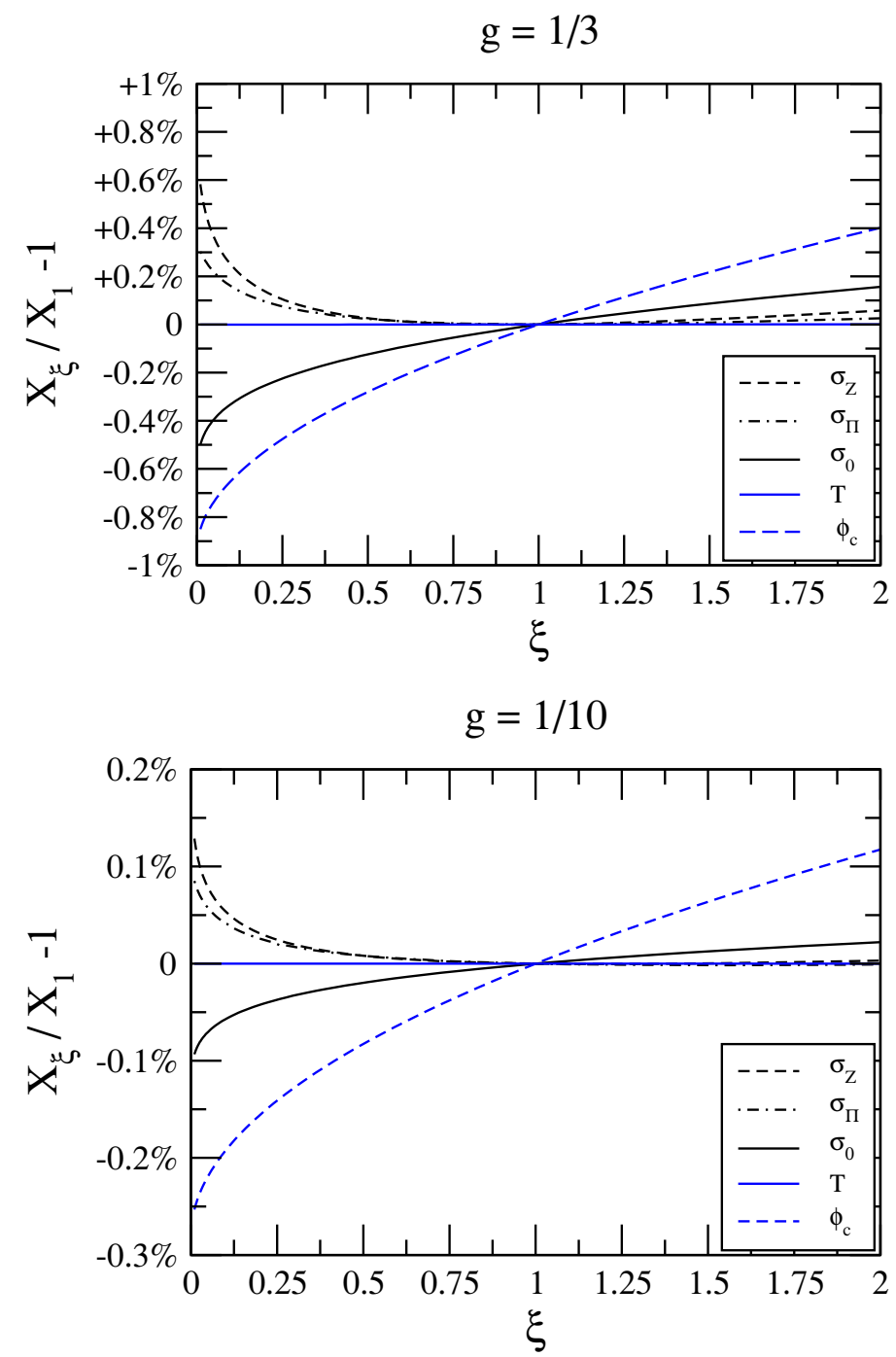

Figure 8. Dependence of the wall tension $\sigma$ on the gauge parameter $\xi$. The three values $\sigma_{0}, \sigma_{Z}$ and $\sigma_{\Pi}$ denote the wall tension deduced, from $V$ only, from $V$ and the naive $Z$ and from the full momentum dependence in $\Pi$, respectively. For comparison, also the critical Temperature $T_{c}$ and the critical field expectation value $\phi_{c}$ are shown. The residual gauge dependence of the wall tension is of relative order $g^{3 / 2} \ln (\xi)$, while for $T_{c}$ it is very small, of order $g^{3}$. In contrast, the $\xi$ dependence of $\phi_{c}$ is expected from the Nielsen identity, see eq. (5.17), and its leading contribution scales as $g \sqrt{\xi}$. The parameters of the upper plot are $g=1 / 3$ and $\lambda=0.015$, while the lower uses $g=1 / 10$ and $\lambda=4.05 \times 10^{-4}$.

is not improved systematically by the inclusion of $Z$. Especially, it is still of order $g^{3 / 2}$. Nevertheless it turns out that a cancellation of the $\xi$-dependent contributions occurs for $\xi$ of order one. These findings are supported by our numerical results that are presented in table 1 and figure 8. Some analytical details of this discussion are given in appendix C.

At this point, we would like to comment on [31] that compared the wall tension in the Landau and Feynman gauges for an effective three dimensional theory. There it was also found that including the $Z$ factor does not lead to a systematic reduction of the gauge 
dependence. Nevertheless, the analysis showed that the gauge-independent contributions from $Z$ where as large as the gauge-dependent ones. This discrepancy compared to our analysis is due to the fact that the Goldstone mass was not resummed what leads to a larger gauge dependence in the effective potential as stressed in section 5 .

The fact that the inclusion of $Z$ does not postpone the gauge dependence of the wall tension to the relative order $g^{2}$ or even $g^{3}$ is related to the breakdown of the gradient expansion in the symmetric phase, which leads to a divergence of $Z$ for $\phi \rightarrow 0$. To avoid this problem, we would like to check the gauge independence of the effective action in the symmetric phase without resorting to the gradient expansion. In order to achieve that, one can expand the effective action in $\phi$ around the symmetric phase and obtains

$$
\Gamma=T \int \frac{d^{3} p}{(2 \pi)^{3}} \phi(p) \Pi(p) \phi(p),
$$

with

$$
\Pi(p)=p^{2}+m_{h}^{2}+\Pi_{2}(p) .
$$

The gauge independence of the effective action (3.6) then implies in leading order

$$
\xi \frac{d \Pi_{2}(p)}{d \xi}=2\left(p^{2}+m_{h}^{2}\right) \frac{d C(p)}{d \phi},
$$

where $C(p)$ is now understood to be expanded in $\phi$ instead of $p$. In the limit $\phi \rightarrow 0$, the only contribution at one-loop order to $\Pi_{2}$ is the last diagram depicted in figure 12 involving the gauge and Goldstone boson and several tadpole diagrams that however have no momentum dependence. Explicit calculation shows

$$
\xi \frac{d \Pi_{2}}{d \xi}=\frac{g^{2} T \xi}{4 \pi}\left[-m_{\chi}+\frac{2\left(p^{2}+m_{\chi}^{2}\right)}{p} \arctan \left(p / m_{\chi}\right)\right],
$$

and $d C / d \phi$ in accordance with (7.18). Notice also that $\Pi_{2}$ is finite in the limit $p \rightarrow 0$ if the limit $\phi \rightarrow 0$ is taken first

$$
\xi \frac{d \Pi_{2}}{d \xi} \simeq \frac{g^{2} T \xi}{4 \pi}\left[m_{\chi}+\frac{8}{3} \frac{p^{2}}{m_{\chi}}\right] .
$$

The mass term agrees hereby with the one derived from the potential (5.15), while the kinetic term does not

$$
Z_{\mathrm{symm}}=1-\left.\frac{\partial \Pi_{2}}{\partial p^{2}}\right|_{p=0}=1-\frac{g^{2} T}{3 \pi m_{\chi}}(2+\xi) .
$$

Since the potential agrees independent from what limit $(\phi \rightarrow 0$ or $p \rightarrow 0)$ is taken first, it is tempting to reduce the gauge dependence of the wall tension by interpolating between the two different kinetic terms $Z$ and $Z_{\text {symm. }}$. In order to implement this idea, we replace the wave function correction in eq. (7.5) by

$$
Z(\phi, p)=1-\frac{\partial \Pi_{2}(\phi, p)}{\partial p^{2}} .
$$




\begin{tabular}{|c|c|c|c|c|c|c|}
\hline & \multicolumn{3}{|c|}{$g=1 / 3$} & \multicolumn{3}{c|}{$g=2 / 3$} \\
\hline$\lambda$ & 0.0075 & 0.015 & 0.025 & 0.06 & 0.1 & 0.15 \\
\hline$\sigma_{0}(\xi=1)$ & 6.29 & 1.78 & 0.74 & 149 & 31.2 & 12.9 \\
\hline$\delta \sigma_{0}$ & $0.11 \%$ & $0.33 \%$ & $0.79 \%$ & $0.33 \%$ & $0.70 \%$ & $1.4 \%$ \\
\hline$\sigma_{Z}(\xi=1)$ & 6.16 & 1.70 & 0.68 & 143 & 28.8 & 11.3 \\
\hline$\delta \sigma_{Z}$ & $-0.10 \%$ & $-0.25 \%$ & $-0.56 \%$ & $-0.21 \%$ & $-0.46 \%$ & $-0.97 \%$ \\
\hline$\sigma_{\Pi}(\xi=1)$ & 6.16 & 1.70 & 0.69 & 143 & 28.8 & 11.3 \\
\hline$\delta \sigma_{\Pi}$ & $-0.07 \%$ & $-0.16 \%$ & $-0.32 \%$ & $-0.13 \%$ & $-0.25 \%$ & $-0.52 \%$ \\
\hline
\end{tabular}

Table 1. Numerical results for the wall tension in units of $10^{-3} v^{3}$, and the shift $\delta \sigma$ for a change of the gauge parameter $\xi$ from 1 to 0.1 . The rows $\sigma_{0}, \sigma_{Z}$ and $\sigma_{\Pi}$ denote the wall tension deduced, from $V$ only, from $V$ and the naive $Z$ and from the full momentum dependence in $\Pi$, respectively.

Here $\Pi_{2}(\phi, p)$ is the full one-loop self-energy for a general expectation value $\phi$ of the Higgs field, which approaches the self-energy $\Pi_{2}(p)$ discussed above for $\phi \rightarrow 0$. On the other hand, $Z(\phi, p \rightarrow 0)$ agrees with the correction to the kinetic term from eq. (6.1). For any non-zero value of the momentum $p, Z(\phi, p)$ has a regular behavior for $\phi \rightarrow 0$, and indeed interpolates between $Z$ and $Z_{\text {symm }}$ as a function of $\phi$ provided that the momentum is chosen small enough, $p \lesssim m_{h}$. In table 1 we show the resulting expression for the wall tension, where we used $p=m_{h} /(2 \pi)$ as the momentum cut-off. Unfortunately, we found that qualitatively the gauge dependence did not improve significantly by doing so, although it is slightly reduced on a quantitative level. A complete cancellation of the gauge dependence at the relative $g^{3 / 2}$ level seems to require the use of the full momentum dependence of the effective action without resorting to the gradient expansion.

\section{Sphaleron numerics}

In this section we briefly discuss the sphaleron energy following [32]. The sphaleron is a static Higgs-gauge configuration that is a saddle point of the action (which reflects the energy of the configuration). It has Chern-Simons number $\frac{1}{2}$ and is situated half-way between two gauge vacua. Our toy model does not contain a $\mathrm{SU}(2)$ gauge sector and hence no sphaleron transitions, but the Higgs potential in the Standard Model has essentially the same features of the Abelian Higgs and it is reasonable to feed the potential (5.15) into the equations of motion of the sphaleron to estimate the gauge dependence of the sphaleron in the Standard Model. In the conventional analysis, the main difference between the Abelian and the non-Abelian model in terms of the strength of the phase transition is that there are three times as many gauge bosons (and ghosts and Goldstones) contributing to the cubic term hence strengthening the phase transition. We will mimic that by also presenting results for larger than observed gauge couplings.

In the non-Abelian case, perturbation theory is plagued by Linde's problem in the symmetric phase such that the expansion in the coupling constant becomes questionable for 


\begin{tabular}{|c|c|c|c|c|c|c|}
\hline & \multicolumn{3}{|c|}{$g=1 / 3$} & \multicolumn{3}{c|}{$g=2 / 3$} \\
\hline$\lambda$ & 0.0075 & 0.015 & 0.025 & 0.06 & 0.1 & 0.15 \\
\hline$\phi_{c} / v$ & 0.965 & 0.549 & 0.325 & 2.07 & 1.03 & 0.535 \\
\hline$T_{c} / v$ & 0.454 & 0.575 & 0.721 & 1.00 & 0.94 & 1.05 \\
\hline$\delta \phi_{c}$ & $0.3 \%$ & $0.7 \%$ & $1.5 \%$ & $0.6 \%$ & $1.3 \%$ & $3.0 \%$ \\
\hline$\delta E_{\mathrm{sph}}$ & $0.01 \%$ & $0.01 \%$ & $0.02 \%$ & $0.05 \%$ & $0.07 \%$ & $0.10 \%$ \\
\hline
\end{tabular}

Table 2. Numerical results for the sphaleron energy. The row $\delta \phi_{c}$ contains the shift for a change of the gauge parameter $\xi$ from 1 to 0.1 . The row contains the corresponding change in sphaleron energy due to a change in the shape of the Higgs potential.

small Higgs vevs. Besides, compared to the tunneling rate discussed in the last section, the convergence of the gradient expansion is even more problematic. The gradient expansion is formally an expansion in the parameter $p^{2} / \mathrm{m}^{2}$ where the relevant mass is the one of the gauge bosons. While for the tunneling bounce this is $\mathcal{O}(g)$, in the case of the sphaleron the expansion parameter is $\mathcal{O}(1)$ and hence not suppressed by any coupling constant even in the broken phase. However, numerically the coefficients $D$ and $Z-1$ are slightly smaller than $C_{0}$ and $V_{\text {eff }}$ (in units of $m_{A}^{2} \phi_{c}^{2}$ ) such that higher orders can be neglected if this trend continues. This issue is to certain extend unrelated to the gauge dependence.

At the same time, the sphaleron energy is proportional to $\phi_{c}$ such that the gauge dependence stemming from $\phi_{c}$ cannot possibly be canceled by the gauge dependence of the effective action solely in the surrounding of the broken phase. In order to quantify our lack of knowledge on $Z$ and $V_{\text {eff }}$ in the symmetric phase, our strategy is to set $Z$ to 1 in the numerical analysis and use the gauge dependence of $V_{\text {eff }}$ to estimate the impact of those contributions to the sphaleron energy.

The differential equations to solve when a spherical Ansatz is used read

$$
\begin{aligned}
\zeta^{2} \frac{d^{2} f}{d \zeta^{2}} & =2 f(1-f)(1-2 f)-\frac{1}{4} \zeta^{2} h^{2}(1-f), \\
\frac{d}{d \zeta} \zeta^{2} \frac{d h}{d \zeta} & =2 h(1-f)^{2}+\frac{1}{g^{2}} \frac{d V_{h}}{d h},
\end{aligned}
$$

with the asymptotic behavior

$$
f \rightarrow \alpha \zeta^{2}, \quad h \rightarrow \beta \zeta \quad \text { for } \quad \zeta \rightarrow 0
$$

and

$$
f \rightarrow 1-\gamma \exp (-\zeta / 2), \quad h \rightarrow 1-\frac{\delta}{\zeta} \exp (-\kappa \zeta) \text { for } \quad \zeta \rightarrow \infty,
$$

where the parameter $\kappa$ is given by $\kappa^{2}=V_{h}^{\prime \prime}$ and the rescaled potential is defined as $V_{h}(h)=$ $V_{\text {eff }}\left(h \cdot \phi_{c}\right)$ while the rescaled coordinate is $\zeta=g \phi_{c}|x|$.

We solve the equations numerically with a shooting algorithm similar to what is used to find the bounce solution of the tunneling action. The parameters $\alpha$ and $\beta$ are chosen and 
the equations are solved from some position $\zeta_{\epsilon}$ close to the origin to a value $\zeta_{\omega} \sim \mathcal{O}(10)$. The parameters $\alpha$ and $\beta$ are then varied and we search for simultaneous zeros in the functions

$$
\begin{aligned}
& D_{f}=1-f+2 f^{\prime}, \\
& D_{h}=\zeta \kappa(1-h)+(\zeta h)^{\prime} .
\end{aligned}
$$

These two conditions ensure that the numerical solutions smoothly match the asymptotic behavior given in (8.3). The sphaleron energy is then given by

$$
\begin{aligned}
E=\frac{4 \sqrt{2} \pi \phi_{c}}{g} \int_{0}^{\infty} d \zeta\left[4\left(\frac{d f}{d \zeta}\right)^{2}+\right. & \frac{8}{\zeta^{2}}(f(1-f))^{2} \\
& \left.+\frac{1}{2} \zeta^{2}\left(\frac{d h}{d \zeta}\right)^{2}+(h(1-f))^{2}+\zeta^{2} g^{-2} V_{h}\right] .
\end{aligned}
$$

Some numerical results are given in table 2. Main impact of the sphaleron energy has the gauge-dependent shift in $\phi_{c}$. The gauge dependence in the critical temperature $T_{c}$ and the shape of the effective potential are subleading. Moreover, close to borderline case of sphaleron washout $\phi_{c} / T_{c} \sim 1$, the uncertainty in the sphaleron energy never exceeds a few percent for Standard Model values of the gauge coupling. In summary, the uncertainty stemming from the residual gauge dependence is subleading compared to corrections coming from two-loop contributions to the effective potential [10,33]. This gauge dependence is inherited from the critical vev

$$
\frac{\xi}{E} \frac{d E}{d \xi} \simeq \frac{g \sqrt{\xi}}{16 \pi \sqrt{2}} \frac{T}{\phi_{c}}
$$

and scales as $g$. To remove this gauge dependence one would probably need to include the next-to-leading order of the kinetic term of the gauge-bosons. There, a sizable gauge dependence is expected in order to ensure a gauge-independent position of the pole in the gauge-boson propagator. Besides, the breakdown of the gradient expansion should lead to even more severe effects than in the case of thermal tunneling as discussed above. This will further complicate the determination of the sphaleron energy with an accuracy beyond the bound (8.6).

\section{Summary}

Let us summarize our findings concerning the gauge dependence of the effective action in the Abelian Higgs model in $R_{\xi}$-gauges. We explicitly demonstrated various Nielsen identities in the regime where the use of perturbation theory and the gradient expansion of the effective action is feasible.

In particular, we have shown that the position of the minimum of the effective potential transforms in leading order according to (3.8) under a change in the gauge fixing parameter $\xi$. We would like to emphasize that this result could only be obtained by calculating the effective potential consistently to order $g^{3}$ using the counting $g^{3} \sim \lambda$. In particular, this required the resummation of contributions to the Goldstone boson mass ${ }^{7}$ of order $m_{A} T$ (where $m_{A}$ denotes collectively the different masses of the gauge bosons).

\footnotetext{
${ }^{7}$ At this point of the analysis, we depart from [34-36] that argue for a large gauge dependence of the effective action.
} 
Furthermore, we have demonstrated that the off-shell effective action in gradient expansion transforms according to the Nielsen identity (4.3). However, this relation cannot guarantee the gauge independence of vacuum transitions for several reasons. First, the gradient expansion is not well justified in these cases since the contribution of the kinetic term to the action is of equal size as (or even larger than) the contribution from the scalar potential. Second, even for small gradients the gradient expansion and the relation (4.3) break down at some point in the symmetric phase and vacuum transitions are also sensitive to this regime. Compared to the analysis at zero temperature [11], notice also the additional contribution involving $\tilde{D}$ that was missing but also not important in the analysis presented there.

Finally, we discussed the gauge dependence of the tunneling action. Using the established procedure to calculate the tunneling action perturbatively (meaning a canonical kinetic term and the appropriate effective potential), we found for the gauge dependence of the wall tension the estimate

$$
\frac{\xi}{\sigma} \frac{d \sigma}{d \xi} \simeq \frac{1}{8 \pi} \frac{m_{\chi} T_{c}}{\phi_{c}^{2}}
$$

what scales as $\sqrt{\lambda} \sim g^{3 / 2}$ (the Goldstone mass $m_{\chi}$ is evaluated in the symmetric phase). Including corrections to the kinetic term $Z$ leads to corrections of the same order but the gauge dependence is not persistently reduced. This is due to the fact that the latter corrections are sensitive to the effective action very close to the symmetric phase where the gradient expansion breaks down.

Since we did not arrive at an explicitly gauge-independent result for $\sigma$, this leaves the question what is the best gauge to chose. Our results develop the strongest $\xi$ dependence for $\xi \sim 0$. For $\xi \gtrsim g$ the wall tension computed using a canonical kinetic term depends logarithmically on $\xi$, while when including the correction to the kinetic term the $\xi$ dependence partially cancels and $\sigma$ is rather insensitive to $\xi$ for $\xi \sim 1$. This could indicate that a $\xi$ value of this order is the appropriate choice and not Landau gauge (that is mostly used in the literature and is reproduced in our case by $\xi \rightarrow 0$ ). Nevertheless, quantitatively the dependence on $\xi$ is rather small and including the two-loop contributions to the effective potential will probably have a larger impact in most models (e.g. contributions from the gluons [37]). We also identified gauge-independent corrections to the wall tension that arise from the kinetic term $Z$ and scale as $g$. Also these contributions are more important than the gauge-dependent ones.

Several non-perturbative studies of tunneling in the Standard Model were presented in refs. [20, 38-41] and a detailed comparison with the perturbative results in Landau gauge was given in [42]. Also there it was concluded that the corrections to the kinetic term and two-loop contributions to the effective potential are important to achieve a good agreement with the non-perturbative results for the wall tension. Numerically, these corrections are far more important than the gauge fixing dependence we discussed here.

In case of the sphaleron, the convergence of the gradient expansion is also problematic. As a naive estimate of the gauge dependence of the sphaleron energy, we obtained

$$
\frac{\xi}{E} \frac{d E}{d \xi} \simeq \frac{g \sqrt{\xi}}{16 \pi \sqrt{2}} \frac{T_{c}}{\phi_{c}}
$$


what is typically of a few percent. Over all, the effective potential enters in the sphaleron energy mostly via the position of its minimum. Hence, improving the gauge dependence of the sphaleron energy will probably require not only to go beyond the gradient expansion but also to calculate the gauge dependence of the wave function corrections of the gauge fields, what we did not attempt here.

The estimates (9.2) and (7.15) are solely based on the Nielsen identity for the Higgs vev, $C_{0} \equiv \xi d \phi / d \xi$. Therefore, the results can be readily carried over to the Standard Model and some of its extensions as the two-Higgs doublet model or singlet extensions. Compared to the Abelian Higgs model, the electroweak sector of these models gives in leading order a gauge fixing dependence of the Higgs vev that is larger by a factor three.

In conclusion, determining vacuum transitions in an explicitly gauge-independent fashion is mostly hindered by the breakdown of the gradient expansion of the effective action (in particular in the vicinity of the symmetric phase). Still, in the cosmologically most interesting regime with $g \ll 1$ and $\phi_{c} / T_{c} \gtrsim 1$, the gauge dependence of the tunneling action and the sphaleron energy is rather small. The situation further improves when the essential cubic contributions to the effective potential do not solely arise from the gauge bosons (as in the present Abelian toy model) but e.g. from additional degrees of freedom (as in the light stop scenario [43-45]) or from the tree level dynamics of an extended scalar sector [46].

\section{Acknowledgments}

We thank W. Buchmüller and M. Laine for helpful discussions. This work has been supported by the German Science Foundation (DFG) within the Collaborative Research Center 676 "Particles, Strings and the Early Universe".

\section{A Feynman rules}

The Feynman rules for the scalar particles are shown in figure 9 while the Feynman rules involving the vector particles are given in figure 10 .

\section{B Wave function corrections}

In this section, we present some results on the coefficient of the kinetic term $Z$ in the $R_{\xi}$-gauge and also for the functions $D$ and $\tilde{D}$. As mentioned in section 2 , derivatives with respect to the background field $\tilde{\phi}$ are in the $R_{\xi}$-gauge not related to diagrams with external Higgs fields. In order to remedy this issue, we replace in the gauge fixing terms the background field $\tilde{\phi}$ by $\tilde{\phi}+\tilde{h} / \sqrt{2}$ where $\tilde{h}$ will be treated as an external field. The function $Z$ is then related to the two-point functions involving $h$ and $\tilde{h}$ via

$$
Z^{\mu \nu}=\frac{\partial^{2}}{\partial p_{\mu} \partial p_{\nu}}\left(\Pi_{h h}+\Pi_{h \tilde{h}}+\Pi_{\tilde{h} h}+\Pi_{\tilde{h} \tilde{h}}\right)
$$



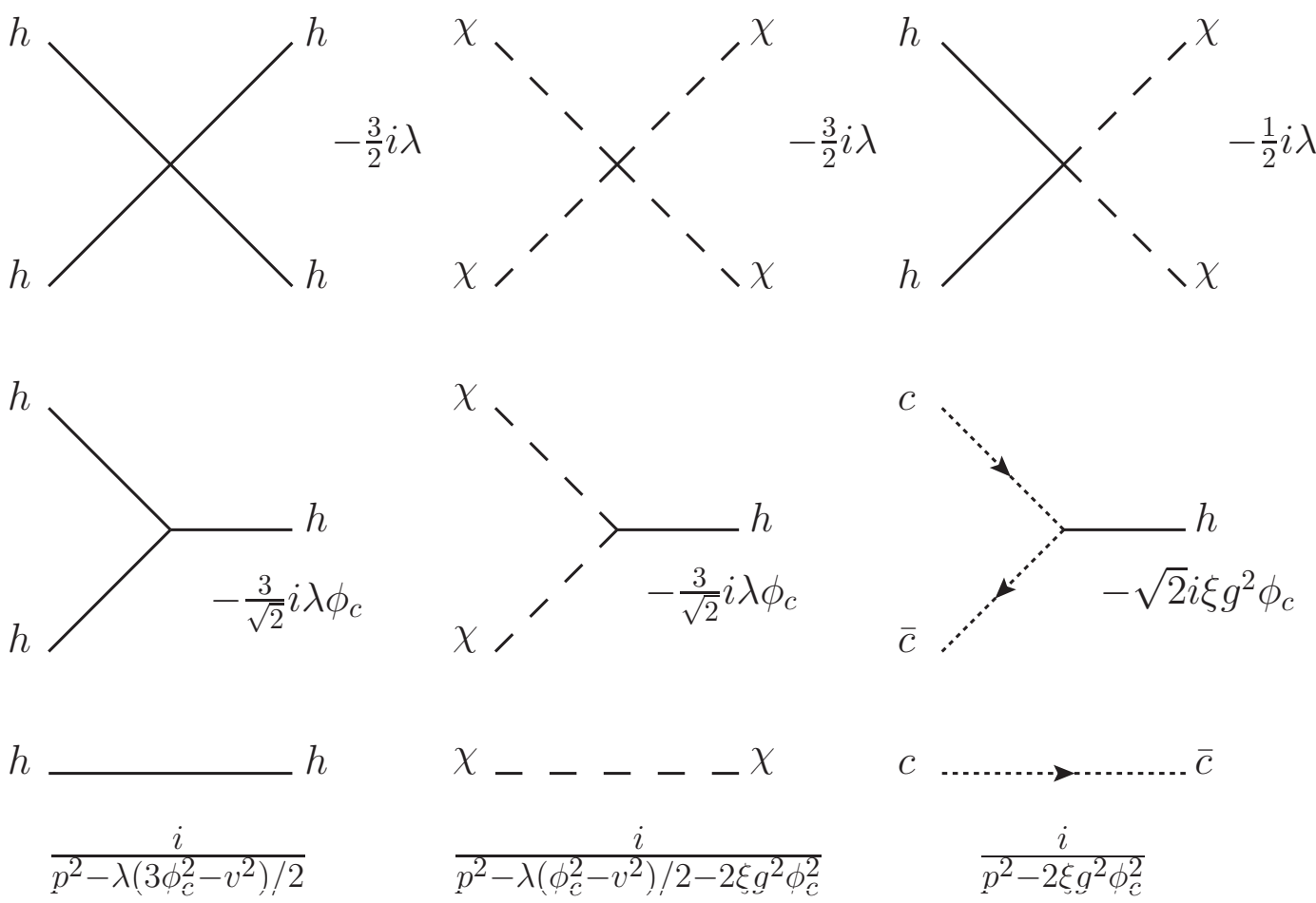

Figure 9. Feynman rules for scalars in the Abelian Higgs model.
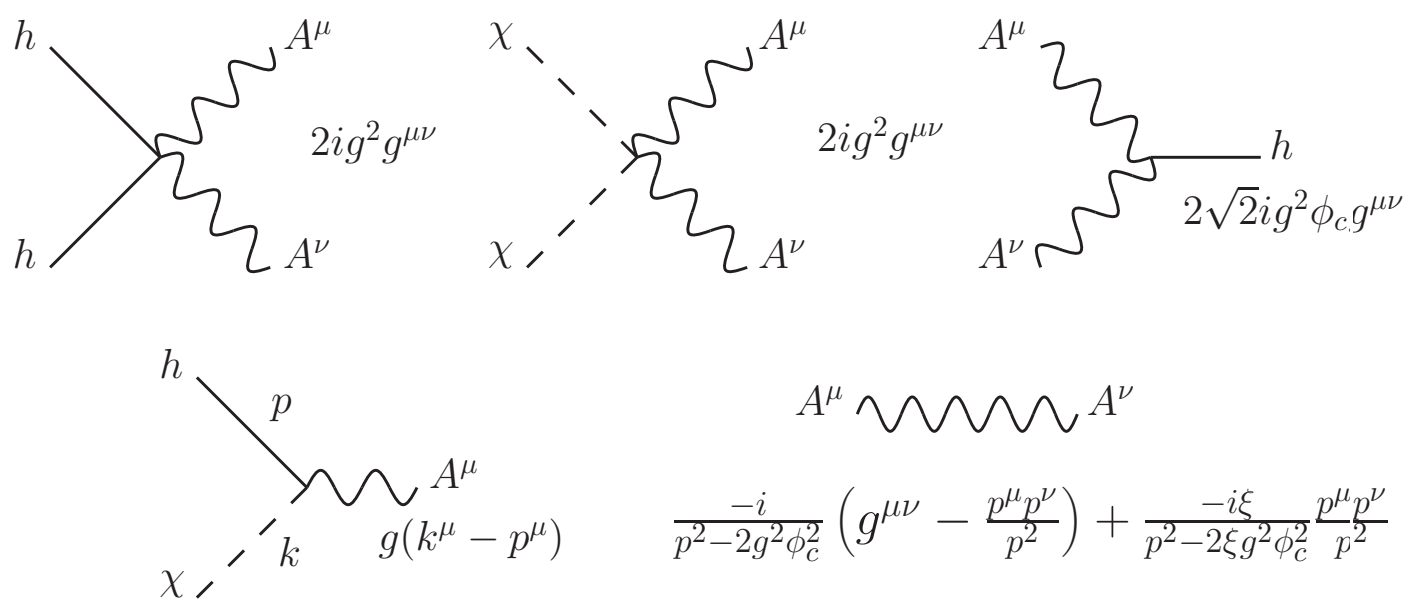

Figure 10. Feynman rules for vectors in the Abelian Higgs model.

The additional Feynman rules involving the external field $\tilde{h}$ are shown in figure 11 . For the sphaleron and thermal tunneling, we only need the spatial components $Z^{i i}$ at finite temperature.

On the one loop level the possible diagrams are of the form depicted in figure 12 where the external lines can be either $h$ or $\tilde{h}$. On one loop level it is more practical to first perform the sum in (B.1) before the integrals are evaluated. The momentum dependence 

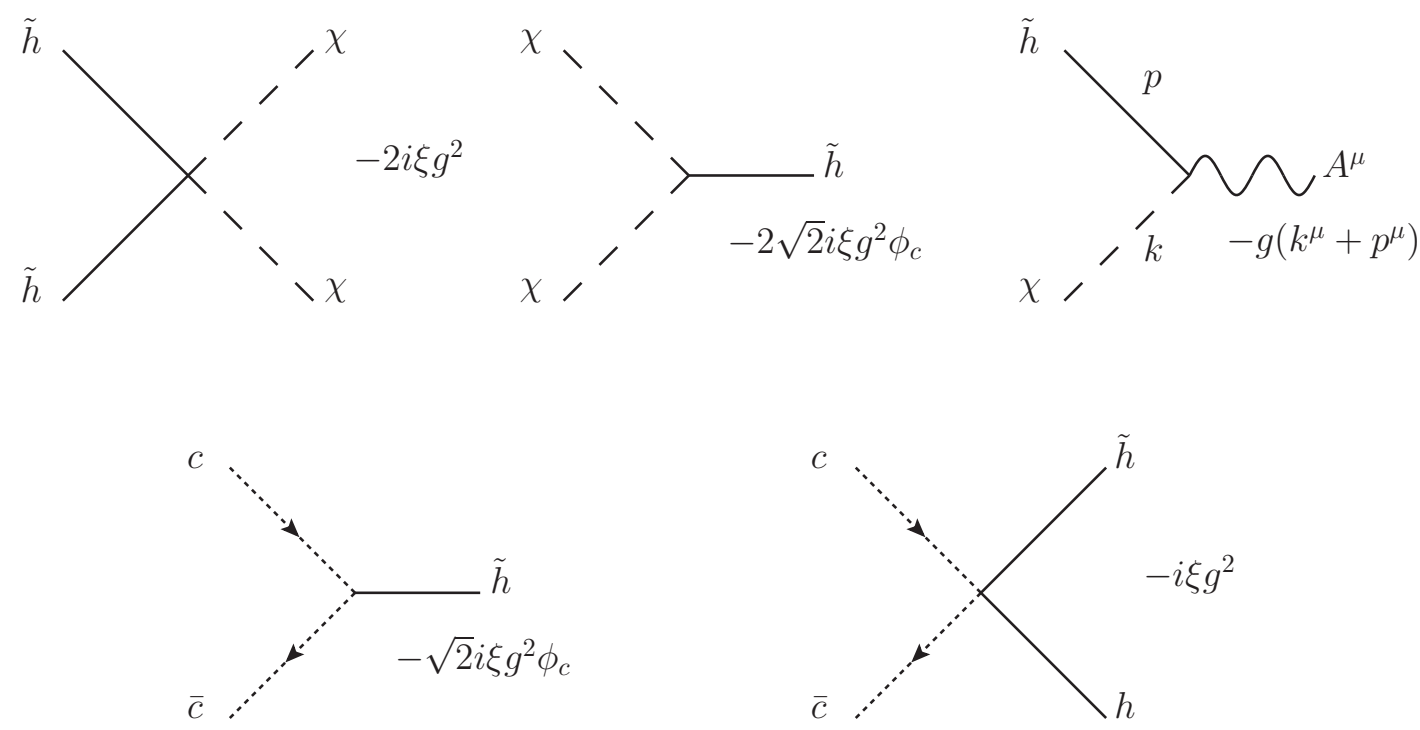

Figure 11. Additional Feynman rules for the kinetic term in the effective action.
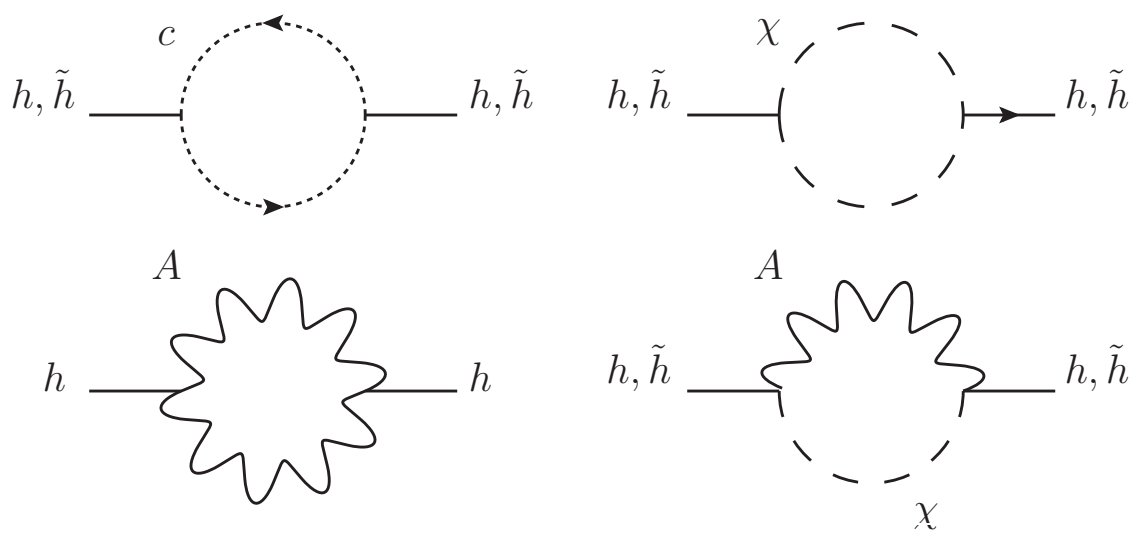

Figure 12. The diagrams contributing to the kinetic term of the effective action.

stems from the integrands

$$
\begin{aligned}
\frac{1}{(l+p / 2)^{2}+m^{2}} \frac{1}{(l-p / 2)^{2}+m^{2}} & \simeq \frac{1}{\left(l^{2}+m^{2}\right)^{2}}-\frac{1}{2} \frac{p^{2}}{\left(l^{2}+m^{2}\right)^{3}}+\frac{(p \cdot l)^{2}}{\left(l^{2}+m^{2}\right)^{4}} \\
& \simeq \frac{1}{\left(l^{2}+m^{2}\right)^{2}}-\frac{1}{6} \frac{p^{2}}{\left(l^{2}+m^{2}\right)^{3}}-\frac{1}{3} \frac{p^{2} m^{2}}{\left(l^{2}+m^{2}\right)^{4}}
\end{aligned}
$$

that can be evaluated using

$$
\begin{aligned}
L_{B}(y) & =\frac{1}{2 \pi^{2}} \sum_{n} \int d x x^{2} \frac{1}{\left(4 \pi^{2} n^{2}+x^{2}+y\right)^{3}} \\
& =\frac{1}{2} \frac{\partial^{2}}{\partial^{2} y} J_{B}(y) \simeq \frac{1}{32 \pi} y^{-3 / 2},
\end{aligned}
$$


and a similar expression for the the integrals with higher powers. Notice that only the zero mode $n=0$ contributes to the leading term and we neglect higher Matsubara modes in the following whenever this holds true.

We first restrict ourselves to the broken phase where the masses are given as

$$
m_{A}^{2}=2 g^{2} \phi^{2} \equiv m^{2} \equiv y T^{2}, \quad m_{\chi}^{2}=m_{\mathrm{FP}}^{2}=\xi m^{2} .
$$

Then one finds for the ghost and Goldstone loops the contributions

$$
\frac{1}{3} g^{2} \xi^{2} y\left(L_{B}(\xi y)+2 \xi y M_{B}(\xi y)\right)
$$

The diagram involving two gauge fields requires the evaluation of products of the polarization projections $P^{\mu \nu}(l)=l^{\mu} l^{\nu} / l^{2}$

$$
\begin{aligned}
P^{\mu \nu}(l+p / 2) P_{\mu \nu}(l-p / 2) & \simeq 1-\frac{p^{2}}{l^{2}}+\frac{(p \cdot l)^{2}}{l^{4}}+\mathcal{O}\left(p^{3}\right) \\
& \simeq 1-\frac{2}{3} \frac{p^{2}}{l^{2}}+\mathcal{O}\left(p^{3}\right) .
\end{aligned}
$$

and again the expansion of the integrand (B.2). The novel integrals are given in appendix D. The contribution from the term involving the $p^{\mu}$ polarization twice gives

$$
\frac{1}{3} g^{2} \xi^{2} y\left(-L_{B}(\xi y)-2 \xi y M_{B}(\xi y)-4 \bar{L}_{B}(\xi y, \xi y, 0)\right) .
$$

The contribution involving one $p^{\mu}$ polarization yields

$$
\frac{8}{3} g^{2} \xi y \bar{L}_{B}(\xi y, y, 0)
$$

and the contribution with no $p^{\mu}$ polarization is

$$
2 g^{2} y\left(-\frac{1}{2} L_{B}(y)-y M_{B}(y)-\frac{2}{3} \bar{L}_{B}(y, y, 0)\right) .
$$

Finally, the contributions from the mixed gauge-Goldstone loop give

$$
4 g^{2} \xi\left(\frac{1}{3} \bar{K}_{B}(\xi y, \xi y)\right),
$$

and

$$
4 g^{2}\left(\frac{2}{3} \bar{K}_{B}(\xi y, y)\right)
$$

In the broken phase, where $m_{\mathrm{FP}}^{2}=m_{\chi}^{2}$, the contributions proportional to $L_{B}$ in (B.5) and (B.7) cancel each other. The third term involving $\bar{L}_{B}$ in (B.7) cancels in leading order against (B.10). Besides, the terms in (B.8) and (B.11) combine to

$$
\frac{8}{3} g^{2}\left(\xi y \bar{L}_{B}(\xi y, y, 0)+\bar{K}_{B}(\xi y, y)\right) \simeq \frac{2 g^{2}}{3 \pi \sqrt{y}}
$$


what is gauge-independent. Together with the contribution in (B.9) this yields the final result

$$
Z \simeq 1-\frac{7 g^{2}}{16 \pi \sqrt{y}}
$$

Taking in addition the resummed gauge boson propagator

$$
\frac{-i}{p^{2}-m_{L}^{2}} P_{\mu \nu}^{L}+\frac{-i}{p^{2}-m_{T}^{2}} P_{\mu \nu}^{T}+\frac{-i}{p^{2}-m_{\mathrm{FP}}^{2}} P_{\mu \nu}^{0}
$$

into account, where $P_{\mu \nu}^{0}=p^{\mu} p^{\nu} / p^{2}, P_{\mu \nu}^{T}=g_{\mu \nu}-u_{\mu} u_{\nu}-\left(\tilde{p}_{\mu} \tilde{p}_{\nu}\right) / \tilde{p}^{2}, P_{\mu \nu}^{L}=g_{\mu \nu}-\left(p_{\mu} p_{\nu}\right) / p^{2}-$ $P_{\mu \nu}^{T}, \tilde{p}_{\mu}=p_{\mu}-u_{\mu}(u p), u_{\mu}=(1,0,0,0), m_{L}^{2}=m_{T}^{2}+a g^{2} T^{2}$, and $m_{T}^{2}=2 g^{2} \phi^{2}$, we find

$$
Z=1-\frac{g^{2}}{48 \pi \sqrt{y}}\left(22-\frac{m_{T}^{3}}{m_{L}^{3}}\right)
$$

The above calculation can be extended to a configuration in field space away from the broken minimum. In that case one cannot set the Goldstone mass $m_{\chi}$ and the mass $m_{\mathrm{FP}}$ of the ghost and of the time-like gauge boson polarization to be equal. In that case we obtain the result given in eq. (6.1)

A similar calculation can be done for the factors $D$ and $\tilde{D}$ in (4.2). These are obtained from calculating the diagrams shown in figure 13 and figure 14, respectively, expanding in powers of the external momentum $p_{\mu}$, and extracting the contribution proportional to $p^{2}$. This yields the result

$$
\begin{aligned}
& D=\frac{g^{2} T \xi}{192 \pi \phi}\left(\frac{1}{m_{\chi}^{3}}+\frac{3 m_{\mathrm{FP}}^{2}}{m_{\chi}^{5}}+\frac{9}{m_{\chi} m_{\mathrm{FP}}\left(m_{\chi}+m_{\mathrm{FP}}\right)}+\frac{8}{\left(m_{\chi}+m_{\mathrm{FP}}\right)^{3}}\right. \\
& -\frac{32 m_{\mathrm{FP}}^{2}}{\xi\left(m_{\chi}^{2}-m_{\mathrm{FP}}^{2}\right)}\left(\frac{1}{m_{\mathrm{FP}}\left(m_{\mathrm{FP}}+m_{T}\right)^{2}}-\frac{1}{m_{\chi}\left(m_{\chi}+m_{T}\right)^{2}}\right) \\
& +\frac{\delta}{\xi}\left[\frac{4 m_{\mathrm{FP}}\left(m_{\chi}+5 m_{\mathrm{FP}}\right)}{m_{\chi}\left(m_{\chi}+m_{\mathrm{FP}}\right)^{4}}+\frac{6 m_{\mathrm{FP}}^{2}}{m_{\chi}^{5}}\right] \\
& \left.+\left(\frac{\delta}{\xi}\right)^{2} \frac{m_{\mathrm{FP}}^{4}\left(25 m_{\chi}^{3}+29 m_{\chi}^{2} m_{\mathrm{FP}}+15 m_{\chi} m_{\mathrm{FP}}^{2}+3 m_{\mathrm{FP}}^{3}\right)}{m_{\chi}^{5}\left(m_{\chi}+m_{\mathrm{FP}}\right)^{5}}\right), \\
& \tilde{D}=-\frac{g^{2} T \xi}{96 \pi}\left(\frac{1}{m_{\chi} m_{\mathrm{FP}}\left(m_{\chi}+m_{\mathrm{FP}}\right)}+\frac{1}{m_{\chi}^{3}}+\frac{4}{\left(m_{\chi}+m_{\mathrm{FP}}\right)^{3}}\right. \\
& \left.+\frac{\delta}{\xi} \frac{m_{\mathrm{FP}}^{2}\left(7 m_{\chi}^{2}+4 m_{\chi} m_{\mathrm{FP}}+m_{\mathrm{FP}}^{2}\right)}{m_{\chi}^{3}\left(m_{\chi}+m_{\mathrm{FP}}\right)^{4}}\right) .
\end{aligned}
$$

\section{Wall tension}

In this section we discuss the gauge dependence of the wall tension in more detail.

First, we show that an explicit calculation reproduces the dependence advocated in (7.11) by use of the Nielsen identity. The wall tension (without including the $Z$ factor) is given by

$$
\sigma_{0}=\int d \phi \sqrt{V_{0}+\delta V}
$$



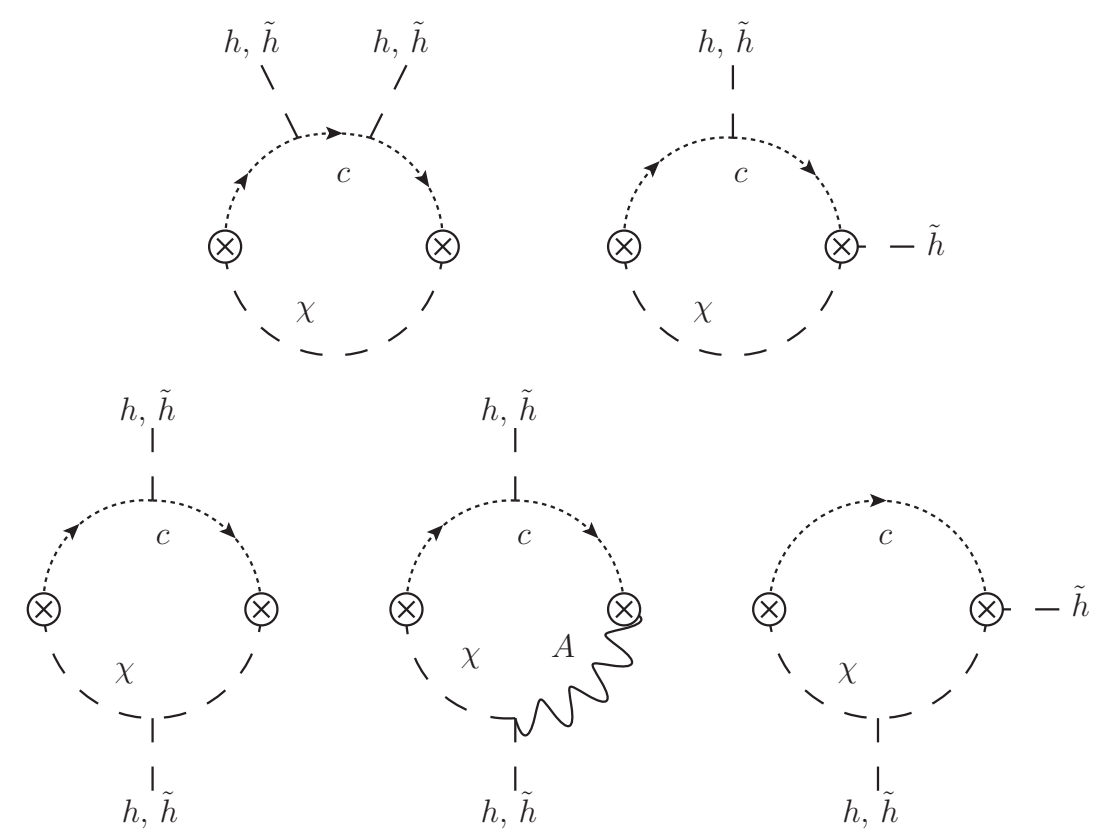

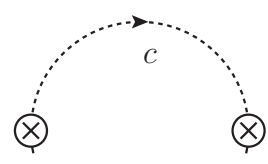

'

$h, \tilde{h} \quad h, \tilde{h}$

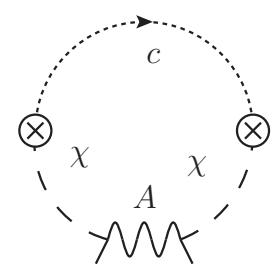

$h, \tilde{h}$
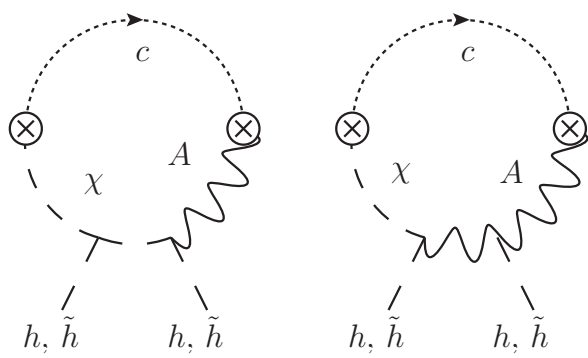

Figure 13. The diagrams contributing to the function $D$.

where we split the potential into a gauge-independent piece $V_{0}$ and the contribution arising from the Goldstone bosons (5.16). The contribution to the wall tension from the broken phase is nearly gauge-independent as long as the integration boundaries are adapted consistently. Close to the symmetric phase, two sources of gauge dependence arises. The first stems from the upper boundary of integration and the second from $\delta V$. Due to $\delta V \ll V_{0}$ one finds

$$
\Delta \sigma_{0}=\frac{1}{2} \int_{0}^{\bar{\phi}} d \phi \frac{\delta V}{\sqrt{V}}+\left.\sqrt{V} C_{0}\right|_{0} ^{\bar{\phi}},
$$

and $\bar{\phi}$ is arbitrary as long as $2 g^{2} \xi \bar{\phi}^{2} \gg m_{\chi}^{2}$. Let us first evaluate this integral in the regime $\bar{\phi} \ll \phi_{c}$. This requires $\xi \gg \lambda / 2 g^{2}$ and leads to $V \simeq m_{\chi}^{2} \phi^{2}$ and $m_{\chi}^{2}=\lambda \phi_{c}^{2} / 4=$ const. The corresponding integral can be evaluated using

$$
\int_{0}^{\bar{x}} \frac{d x}{x}\left(\left(1+x^{2}\right)^{3 / 2}-x^{3}-1\right) \equiv \frac{3}{2} \bar{x}-\log (\bar{x})-\frac{4}{3}+\log 2+\mathcal{O}(\log (\bar{x}) / \bar{x})
$$




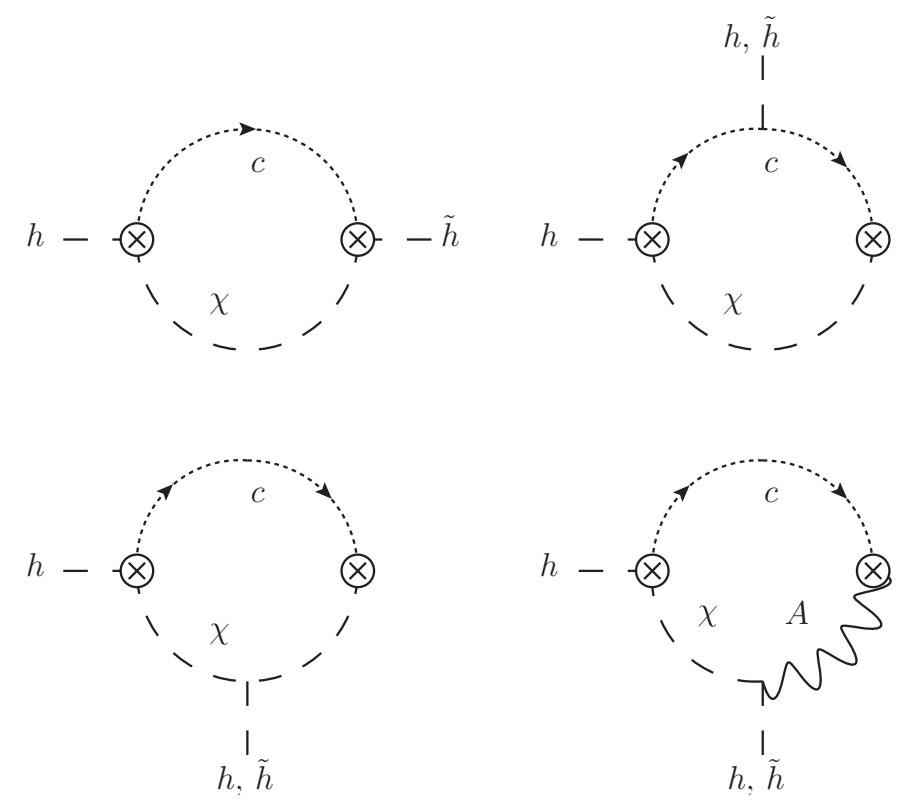

Figure 14. The diagrams contributing to the function $\tilde{D}$.

We are mostly interested in the two leading terms. The constant term do not lead to a $\xi$ dependence and the subleading corrections are of order $\lambda \log (g) / g^{2} \xi \sim g \log (g) / \xi$. In the wall tension, one cannot chose $\bar{\phi}$ parametrically smaller than $\phi_{c}$, but one can neglect the range of integration from zero to a few, since it does not contribute to the two leading terms. This makes the result meaningful, since the effective action cannot be trusted for $x \ll 1$ due to the breakdown of the gradient expansion. This also allows one to expand the numerator of the integrand and to include the full mean field potential and not just its linearization in the symmetric phase. The arising integral is

$$
\begin{aligned}
\int_{0}^{\bar{x}} \frac{d x}{x\left(1-x / x_{c}\right)} & \left(\frac{3}{2} x\left(1-x / x_{c}\right)\left(1-2 x / x_{c}\right)-1\right) \\
& \equiv \frac{3}{2} \bar{x}\left(1-\bar{x} / x_{c}\right)-\log (\bar{x})+\log \left(1-\bar{x} / x_{c}\right)+\operatorname{const}+\mathcal{O}(\log (\bar{x}) / \bar{x}) .
\end{aligned}
$$

After this procedure, $\bar{x}$ can be chosen as a fixed multiple of $x_{c}$, e.g. $\bar{x}=x_{c} / 2=$ $\sqrt{2 g^{2} \xi / \lambda}$. The linear term cancels then against the contribution from the boundary of integration (what can be checked using (5.23)) while the logarithmic term reproduces the relation $(7.11)$

$$
\Delta \sigma_{0} \simeq \frac{1}{48 \pi} T_{c} m_{\chi}^{2} \log (\xi) .
$$

The constant term is gauge-independent and corrections to this relation are of relative order $g \log (g) / \xi$.

Next, we examine if the logarithmic terms cancels against the contributions arising from the wave function corrections $Z$. For larger values of $x$, the integrand can be expand as

$$
\Delta \sigma_{Z}=\frac{1}{2} \int_{0}^{\bar{\phi}} d \phi \delta Z \sqrt{V}
$$


This integral still diverges, but one can extract a gauge-independent contribution

$$
Z-1 \stackrel{\phi \rightarrow \infty}{\longrightarrow} \frac{g^{2} T}{3 \pi}\left(\frac{11}{8 m_{T}}-\frac{m_{T}^{2}}{16 m_{L}^{3}}\right)
$$

We will deal with this contribution later.

Since the leading contributions from the remaining $\delta Z$ is of order $\log (\bar{x})$, the potential can be linearized from the start and the integration of the $\xi$-dependent terms of $\delta Z$ in (6.1) leads for large $\bar{x}$ to a contribution

$$
\Delta \sigma_{Z} \simeq-\frac{1}{12 \pi} T_{c} m_{\chi}^{2}\left(-\frac{5}{12}+\frac{4}{1-\xi}+\frac{4 \log (1-\sqrt{1-\xi})-2 \log (\xi)}{(1-\xi)^{3 / 2}}\right)
$$

Note that the right-hand side is regular for $\xi>0$, especially it has no pole for $\xi=1$. It is also possible to estimate the $\xi$ dependence by evaluating the $\xi$-derivative similarly to eq. (7.7), but including $Z$ and using the Nielsen identity eq. (4.3). This leads to the same result as shown above. For $\xi$ not too small, the $\xi$ dependence of $\Delta \sigma_{0}$ and $\Delta \sigma_{Z}$ are of the same order and have opposite signs. However, there is no systematic cancellation between the $\xi$ dependences of these two quantities. In any case, it turns out that it is not justified to neglect the integration in $x$ from zero to a few, since the corresponding contribution is potentially as large as the one we just presented. This contribution cannot reliably determined due to the breakdown of the gradient expansion close to the symmetric phase. Ultimately this prevents us from obtaining a gauge-independent result for the wall tension.

Finally, consider the gauge-independent piece (C.7). The corresponding contribution to the wall tension can be evaluated using

$$
\int_{0}^{1}\left(\sqrt{1-\frac{\alpha x^{2}}{\left(x^{2}+\beta\right)^{3 / 2}}}-1\right) x(1-x) \simeq \begin{cases}\frac{1}{4} \alpha & \text { for } \beta=0 \\ 0.0146 \alpha & \text { for } \beta \simeq 1\end{cases}
$$

Hence, the longitudinal gauge bosons contribute via the wave function correction a term

$$
\Delta \sigma \simeq-\frac{11 \sqrt{2}}{192 \pi} g m_{\chi} T_{c} \phi_{c}
$$

to the wall tension. Notice that this is of order $g^{5 / 2}$ and parametrically larger than the gauge-dependent contributions we estimated before.

\section{Integrals}

In the following we list the used one-loop integrals.

$$
\begin{aligned}
I_{B}(y) & =\frac{1}{2 \pi^{2}} \sum_{n} \int d x x^{2} \log \left(4 \pi^{2} n^{2}+x^{2}+y\right) \\
& \simeq \text { const }+\frac{1}{12} y-\frac{1}{6 \pi} y^{3 / 2}+\mathcal{O}\left(y^{2} \log y\right)
\end{aligned}
$$




$$
\begin{aligned}
J_{B}(y) & =\frac{1}{2 \pi^{2}} \sum_{n} \int d x x^{2} \frac{1}{4 \pi^{2} n^{2}+x^{2}+y} \\
& =\frac{d}{d y} I_{B}(y) \simeq-\frac{1}{4 \pi} y^{1 / 2}+\mathcal{O}(y \log y) \\
\bar{K}_{B}\left(y_{1}, y_{2}\right) & =\frac{1}{2 \pi^{2}} \int d x x^{2} \frac{1}{x^{2}+y_{1}} \frac{1}{x^{2}+y_{2}} \\
& \simeq \frac{1}{4 \pi} \frac{1}{\sqrt{y_{1}}+\sqrt{y_{2}}}+\mathcal{O}(\log y) \\
\bar{L}_{B}\left(y_{1}, y_{2}, y_{3}\right) & =\frac{1}{2 \pi^{2}} \int d x x^{2} \frac{1}{x^{2}+y_{1}} \frac{1}{x^{2}+y_{2}} \frac{1}{x^{2}+y_{3}} \\
& \simeq \frac{1}{4 \pi} \frac{1}{\left(\sqrt{y_{1}}+\sqrt{y_{2}}\right)\left(\sqrt{y_{1}}+\sqrt{y_{3}}\right)\left(\sqrt{y_{3}}+\sqrt{y_{2}}\right)}+\mathcal{O}\left(y^{-1}\right), \\
L_{B}(y) & =\bar{L}_{B}(y, y, y), \\
M_{B}(y) & =\frac{1}{2 \pi^{2}} \int d x x^{2} \frac{1}{\left(x^{2}+y_{1}\right)^{4}} \simeq \frac{1}{64 \pi} y^{5 / 2}+\mathcal{O}\left(y^{-2}\right) .
\end{aligned}
$$

Open Access. This article is distributed under the terms of the Creative Commons Attribution License which permits any use, distribution and reproduction in any medium, provided the original author(s) and source are credited.

\section{References}

[1] S.R. Coleman and E.J. Weinberg, Radiative corrections as the origin of spontaneous symmetry breaking, Phys. Rev. D 7 (1973) 1888 [INSPIRE].

[2] L. Dolan and R. Jackiw, Symmetry behavior at finite temperature, Phys. Rev. D 9 (1974) 3320 [InSPIRE].

[3] R. Jackiw, Functional evaluation of the effective potential, Phys. Rev. D 9 (1974) 1686 [INSPIRE].

[4] L. Dolan and R. Jackiw, Gauge invariant signal for gauge symmetry breaking, Phys. Rev. D 9 (1974) 2904 [inSPIRE].

[5] N. Nielsen, On the gauge dependence of spontaneous symmetry breaking in gauge theories, Nucl. Phys. B 101 (1975) 173 [inSPIRE].

[6] R. Fukuda and T. Kugo, Gauge invariance in the effective action and potential, Phys. Rev. D 13 (1976) 3469 [inSPIRE].

[7] I. Aitchison and C. Fraser, Gauge invariance and the effective potential, Annals Phys. 156 (1984) 1 [nSPIRE].

[8] R. Kobes, G. Kunstatter and A. Rebhan, Gauge dependence identities and their application at finite temperature, Nucl. Phys. B 355 (1991) 1 [InSPIRE].

[9] W. Buchmüller, Z. Fodor and A. Hebecker, Gauge invariant treatment of the electroweak phase transition, Phys. Lett. B 331 (1994) 131 [hep-ph/9403391] [INSPIRE].

[10] W. Buchmüller, Z. Fodor and A. Hebecker, Thermodynamics of the electroweak phase transition, Nucl. Phys. B 447 (1995) 317 [hep-ph/9502321] [INSPIRE]. 
[11] D. Metaxas and E.J. Weinberg, Gauge independence of the bubble nucleation rate in theories with radiative symmetry breaking, Phys. Rev. D 53 (1996) 836 [hep-ph/9507381] [INSPIRE].

[12] K. Fujikawa, B. Lee and A. Sanda, Generalized renormalizable gauge formulation of spontaneously broken gauge theories, Phys. Rev. D 6 (1972) 2923 [INSPIRE].

[13] S. Weinberg, Perturbative calculations of symmetry breaking, Phys. Rev. D 7 (1973) 2887 [INSPIRE].

[14] M. Laine, The two loop effective potential of the $3 D \mathrm{SU}(2)$ Higgs model in a general covariant gauge, Phys. Lett. B 335 (1994) 173 [hep-ph/9406268] [INSPIRE].

[15] M. Laine, Gauge dependence of the high temperature two loop effective potential for the Higgs field, Phys. Rev. D 51 (1995) 4525 [hep-ph/9411252] [INSPIRE].

[16] J. Kripfganz, A. Laser and M.G. Schmidt, The high temperature two loop effective potential of the electroweak theory in a general 't Hooft background gauge, Phys. Lett. B 351 (1995) 266 [hep-ph/9501317] [INSPIRE].

[17] M. Shaposhnikov, Possible appearance of the baryon asymmetry of the universe in an electroweak theory, JETP Lett. 44 (1986) 465 [Pisma Zh. Eksp. Teor. Fiz. 44 (1986) 364] [INSPIRE].

[18] G.W. Anderson and L.J. Hall, The electroweak phase transition and baryogenesis, Phys. Rev. D 45 (1992) 2685 [inSPIRE].

[19] P.B. Arnold and O. Espinosa, The Effective potential and first order phase transitions: Beyond leading-order, Phys. Rev. D 47 (1993) 3546 [Erratum ibid. D 50 (1994) 6662] [hep-ph/9212235] [INSPIRE].

[20] K. Kajantie, M. Laine, K. Rummukainen and M.E. Shaposhnikov, The electroweak phase transition: a nonperturbative analysis, Nucl. Phys. B 466 (1996) 189 [hep-lat/9510020] [INSPIRE].

[21] M. Carrington, The effective potential at finite temperature in the standard model, Phys. Rev. D 45 (1992) 2933 [INSPIRE].

[22] U. Kraemmer, A. Rebhan and H. Schulz, Resummations in hot scalar electrodynamics, Annals Phys. 238 (1995) 286 [hep-ph/9403301] [INSPIRE].

[23] E. Braaten and R.D. Pisarski, Soft amplitudes in hot gauge theories: a general analysis, Nucl. Phys. B 337 (1990) 569 [inSPIRE].

[24] W. Buchmüller, Z. Fodor, T. Helbig and D. Walliser, The weak-electroweak phase transition, Annals Phys. 234 (1994) 260 [hep-ph/9303251] [INSPIRE].

[25] W. Buchmüller and O. Philipsen, Abelian versus non-abelian Higgs model in three-dimensions, Phys. Lett. B 354 (1995) 403 [hep-ph/9504278] [INSPIRE].

[26] A. Hebecker, The electroweak phase transition, hep-ph/9506418 [INSPIRE].

[27] D. Bödeker, W. Buchmüller, Z. Fodor and T. Helbig, Aspects of the cosmological electroweak phase transition, Nucl. Phys. B 423 (1994) 171 [hep-ph/9311346] [INSPIRE].

[28] J. Langer, Statistical theory of the decay of metastable states, Annals Phys. 54 (1969) 258 [INSPIRE].

[29] S.R. Coleman, The fate of the false vacuum. 1. Semiclassical theory, Phys. Rev. D 15 (1977) 2929 [Erratum ibid. D 16 (1977) 1248] [INSPIRE]. 
[30] A.D. Linde, Fate of the false vacuum at finite temperature: theory and applications, Phys. Lett. B 100 (1981) 37 [InSPIRE].

[31] J. Kripfganz, A. Laser and M.G. Schmidt, Perturbative contributions to the electroweak interface tension, Z. Phys. C 73 (1997) 353 [hep-ph/9512340] [INSPIRE].

[32] F.R. Klinkhamer and N. Manton, A saddle point solution in the Weinberg-Salam theory, Phys. Rev. D 30 (1984) 2212 [inSPIRE].

[33] Z. Fodor and A. Hebecker, Finite temperature effective potential to order $g^{4}, \lambda^{2}$ and the electroweak phase transition, Nucl. Phys. B 432 (1994) 127 [hep-ph/9403219] [INSPIRE].

[34] H.H. Patel and M.J. Ramsey-Musolf, Baryon washout, electroweak phase transition and perturbation theory, JHEP 07 (2011) 029 [arXiv:1101.4665] [INSPIRE].

[35] C. Wainwright, S. Profumo and M.J. Ramsey-Musolf, Gravity waves from a cosmological phase transition: gauge artifacts and Daisy resummations, Phys. Rev. D 84 (2011) 023521 [arXiv: 1104.5487] [INSPIRE].

[36] C.L. Wainwright, S. Profumo and M.J. Ramsey-Musolf, Phase transitions and gauge artifacts in an abelian Higgs plus singlet model, arXiv: 1204.5464 [INSPIRE].

[37] J.E. Bagnasco and M. Dine, Some two loop corrections to the finite temperature effective potential in the electroweak theory, Phys. Lett. B 303 (1993) 308 [hep-ph/9212288] [INSPIRE].

[38] Z. Fodor et al., Numerical simulations and the strength of the electroweak phase transition, Phys. Lett. B 334 (1994) 405 [hep-lat/9405021] [INSPIRE].

[39] Z. Fodor, J. Hein, K. Jansen, A. Jaster and I. Montvay, Simulating the electroweak phase transition in the SU(2) Higgs model, Nucl. Phys. B 439 (1995) 147 [hep-lat/9409017] [INSPIRE].

[40] F. Csikor, Z. Fodor, J. Hein and J. Heitger, Interface tension of the electroweak phase transition, Phys. Lett. B 357 (1995) 156 [hep-lat/9506029] [INSPIRE].

[41] F. Csikor, Z. Fodor, J. Hein, A. Jaster and I. Montvay, Numerical tests of the electroweak phase transition and thermodynamics of the electroweak plasma, Nucl. Phys. B 474 (1996) 421 [hep-lat/9601016] [INSPIRE].

[42] G.D. Moore and K. Rummukainen, Electroweak bubble nucleation, nonperturbatively, Phys. Rev. D 63 (2001) 045002 [hep-ph/0009132] [INSPIRE].

[43] M.S. Carena, M. Quirós and C. Wagner, Opening the window for electroweak baryogenesis, Phys. Lett. B 380 (1996) 81 [hep-ph/9603420] [INSPIRE].

[44] D. Delepine, J. Gerard, R. Gonzalez Felipe and J. Weyers, A light stop and electroweak baryogenesis, Phys. Lett. B 386 (1996) 183 [hep-ph/9604440] [INSPIRE].

[45] M.S. Carena, M. Quirós and C. Wagner, Electroweak baryogenesis and Higgs and stop searches at LEP and the Tevatron, Nucl. Phys. B 524 (1998) 3 [hep-ph/9710401] [INSPIRE].

[46] J.R. Espinosa, T. Konstandin and F. Riva, Strong electroweak phase transitions in the standard model with a singlet, Nucl. Phys. B 854 (2012) 592 [arXiv:1107.5441] [INSPIRE]. 